\title{
The AGILE Mission
}

M. Tavani ${ }^{1,2,3}$, G. Barbiellini ${ }^{3,4,5}$, A. Argan ${ }^{1}$, F. Boffelli $^{13}$, A. Bulgarelli ${ }^{8}$, P. Caraveo $^{6}$, P. W. Cattaneo ${ }^{13}$, A. W. Chen $^{3,6}$, V. Cocco $^{2}$, E. Costa ${ }^{1}$, F. D’Ammando ${ }^{1,2}$, E. Del Monte ${ }^{1}$, G. De Paris ${ }^{1}$, G. Di Cocco ${ }^{8}$, G. Di Persio ${ }^{1}$, I. Donnarumma ${ }^{1}$, Y. Evangelista ${ }^{1}$, M. Feroci ${ }^{1}$, A. Ferrari ${ }^{3,16}$, M. Fiorini ${ }^{6}$, F. Fornari ${ }^{6}$, F. Fuschino ${ }^{8}$, T. Froysland ${ }^{3,7}$, M. Frutti ${ }^{1}$, M. Galli ${ }^{9}$, F. Gianotti ${ }^{8}$, A. Giuliani ${ }^{3,6}$, C. Labanti ${ }^{8}$, I. Lapshov ${ }^{1,15}$, F. Lazzarotto ${ }^{1}$, F. Liello ${ }^{5}$, P. Lipari ${ }^{10,11}$, F. Longo ${ }^{4,5}$,

E. Mattaini ${ }^{6}$, M. Marisaldi ${ }^{8}$, M. Mastropietro ${ }^{24}$, A. Mauri ${ }^{8}$, F. Mauri ${ }^{13}$, S. Mereghetti ${ }^{6}$, E. Morelli ${ }^{8}$, A. Morselli ${ }^{7}$, L. Pacciani ${ }^{1}$, A. Pellizzoni ${ }^{6}$, F. Perotti ${ }^{6}$, G. Piano ${ }^{1}$, P. Picozza ${ }^{2,7}$, C. Pontoni ${ }^{3,5}$, G. Porrovecchio ${ }^{1}$, M. Prest $^{5}$, G. Pucella ${ }^{1}$, M. Rapisarda ${ }^{12}$, A. Rappoldi ${ }^{13}$, E. Rossi ${ }^{8}$, A. Rubini ${ }^{1}$, P. Soffitta ${ }^{1}$, A. Traci ${ }^{8}$, M. Trifoglio $^{8}$, A. Trois $^{1}$, E. Vallazza ${ }^{5}$, S. Vercellone ${ }^{6}$, V. Vittorini ${ }^{1,3}$, A. Zambra ${ }^{3,6}$, D. Zanello ${ }^{10,11}$, C. Pittori ${ }^{14}$, B. Preger ${ }^{14}$, P. Santolamazza ${ }^{14}$, F. Verrecchia ${ }^{14}$, P. Giommi ${ }^{14}$, S. Colafrancesco ${ }^{14}$, A. Antonelli ${ }^{17}$, S. Cutini ${ }^{14}$, D. Gasparrini ${ }^{14}$, S. Stellato ${ }^{14}$,

G. Fanari ${ }^{14}$, R. Primavera ${ }^{14}$, F. Tamburelli ${ }^{14}$, F. Viola ${ }^{18}$, G. Guarrera ${ }^{18}$, L. Salotti ${ }^{18}$, F. D' Amico ${ }^{18}$, E. Marchetti $^{18}$, M. Crisconio ${ }^{18}$, P. Sabatini ${ }^{19}$, G. Annoni ${ }^{19}$, S. Alia ${ }^{19}$, A. Longoni ${ }^{19}$, R. Sanquerin ${ }^{19}$, M. Battilana ${ }^{19}$, P. Concari ${ }^{19}$ ', E. Dessimone ${ }^{19}$, R. Grossi ${ }^{19}$, A. Parise ${ }^{19}$, F. Monzani ${ }^{20}$, E. Artina ${ }^{20}$, R. Pavesi ${ }^{20}$, G. Marseguerra ${ }^{20}$, L. Nicolini $^{20}$, L. Scandelli ${ }^{20}$, L. Soli ${ }^{20}$, V. Vettorello ${ }^{20}$, E. Zardetto ${ }^{20}$, A. Bonati ${ }^{20}$, L. Maltecca ${ }^{20}$, E. D’Alba ${ }^{20}$, M. Patané $^{20}$, G. Babini ${ }^{21}$, F. Onorati ${ }^{21}$, L. Acquaroli ${ }^{21}$, M. Angelucci ${ }^{21}$, B. Morelli ${ }^{21}$, C. Agostara ${ }^{21}$, M. Cerone ${ }^{22}$, A. Michetti 22 , P. Tempesta ${ }^{22}$, S. D’Eramo ${ }^{22}$, F. Rocca ${ }^{22}$, F. Giannini ${ }^{22}$, G. Borghi ${ }^{23}$, B. Garavelli ${ }^{25}$, M. Conte ${ }^{20}$, M. Balasini ${ }^{20}$, I. Ferrario ${ }^{25}$, M. Vanotti ${ }^{25}$, E. Collavo ${ }^{25}$, and M. Giacomazzo 25

(Affiliations can be found after the references)

Received 4 July 2008 / Accepted 27 November 2008

\section{ABSTRACT}

Context. AGILE is an Italian Space Agency mission dedicated to observing the gamma-ray Universe. The AGILE's very innovative instrumentation for the first time combines a gamma-ray imager (sensitive in the energy range $30 \mathrm{MeV}-50 \mathrm{GeV}$ ), a hard X-ray imager (sensitive in the range $18-60 \mathrm{keV}$ ), a calorimeter (sensitive in the range $350 \mathrm{keV}-100 \mathrm{MeV}$ ), and an anticoincidence system. AGILE was successfully launched on 2007 April 23 from the Indian base of Sriharikota and was inserted in an equatorial orbit with very low particle background.

Aims. AGILE provides crucial data for the study of active galactic nuclei, gamma-ray bursts, pulsars, unidentified gamma-ray sources, galactic compact objects, supernova remnants, $\mathrm{TeV}$ sources, and fundamental physics by microsecond timing.

Methods. An optimal sky angular positioning (reaching 0.1 degrees in gamma-rays and 1-2 arcmin in hard X-rays) and very large fields of view ( $2.5 \mathrm{sr}$ and $1 \mathrm{sr}$, respectively) are obtained by the use of Silicon detectors integrated in a very compact instrument.

Results. AGILE surveyed the gamma-ray sky and detected many Galactic and extragalactic sources during the first months of observations. Particular emphasis is given to multifrequency observation programs of extragalactic and galactic objects.

Conclusions. AGILE is a successful high-energy gamma-ray mission that reached its nominal scientific performance. The AGILE Cycle-1 pointing program started on 2007 December 1, and is open to the international community through a Guest Observer Program.

Key words. instrumentation: detectors - techniques: high angular resolution - techniques: image processing gamma rays: observations - X-rays: general

\section{Introduction}

Gamma-ray astrophysics above $100 \mathrm{MeV}$ is an exciting field of astronomical sciences that has received a strong impulse in recent years. Detecting cosmic gamma-ray emission in the energy range from a few tens of $\mathrm{MeV}$ to a few tens of $\mathrm{GeV}$ is only possible from space instrumentation, and in the past 20 years, several space missions have confronted the challenge of detecting cosmic gamma-rays. Gamma-ray emission from cosmic sources at these energies is intrinsically non-thermal, and provides a diagnostic of particle acceleration and radiation processes in extreme conditions. Gamma-ray sources showing the most energetic phenomena of our Universe include: Galactic compact objects and related sources (e.g., black hole binaries, pulsars, SNRs, pulsar wind nebulae, binary systems with accreting neutron stars, and colliding wind binaries), Galactic molecular clouds shining in gamma-rays because of their interaction with energetic cosmic rays, extragalactic massive black holes residing in active galactic nuclei (AGN), exploding massive stars producing gamma-ray bursts (GRBs).

The history of gamma-ray astronomy is a topic beyond the scope of this paper. We briefly summarize here the pioneering efforts and experiments that were carried out over the years. The first detection of cosmic energetic radiation of energy well above the electron's rest mass started in the late Sixties with the detection of gamma-rays above $50 \mathrm{MeV}$ by the OSO-3 satellite (Kraushaar et al. 1972). The first gamma-ray telescope with an angular resolution of a few degrees was launched in 1972 November by the Malindi site in Kenya as the second Small Astronomy Satellite (SAS-2). A clear concentration of gammaray emission from the Galactic plane was firmly established, together with a handful of pointlike gamma-ray sources (Fichtel et al. 1975; Bignami et al. 1979). The European mission COS-B 
was launched on 1975 August 8 and after 7 years of observations several gamma-ray sources were studied and catalogued (Mayer-Hasselwander et al. 1979; Swanenburg et al. 1981).

The Compton Gamma-Ray Observatory (CGRO) (active between 1991-2000) substantially increased our knowledge of the gamma-ray Universe and provided a wealth of data on a wide variety of sources, as well as unsolved puzzles. In particular, CGRO hosted the Energetic Gamma-Ray Experiment Telescope (EGRET) (operating in the energy range $30 \mathrm{MeV}-30 \mathrm{GeV}$ ) which carried out a complete sky survey that detected hundreds of gamma-ray sources (Fichtel \& Trombka 1997; Hartman et al. 1999; Thompson et al. 1993, 1995, 1996, 1998).

The EGRET scientific inheritance is the starting point for any high-energy mission exploring the Universe above $100 \mathrm{MeV}$. The current new generation of gamma-ray space missions has to address some of the fundamental issues that were left open (or unresolved) by EGRET. It is then important to make substantial progress in the instrumentation and mission concepts to achieve the following goals: (1) improving the gamma-ray angular resolution near $100 \mathrm{MeV}$ by at least a factor of 2-3 compared to EGRET; (2) obtaining the largest possible field of view (FOV) at $100 \mathrm{MeV}$ reaching $2.5-3 \mathrm{sr}$, (3) drastically reducing the deadtime for gamma-ray detection from the EGRET value of $100 \mathrm{~ms}$, (4) obtaining broadband spectral information possibly including a simultaneous detection capability in the MeV and X-ray energy ranges; (5) carrying out a rapid quicklook analysis of the gamma-ray data and a fast dissemination of results and alerts; (6) stimulating efficient multifrequency programs. The AGILE Mission is aimed at meeting all these goals with an unprecedented configuration for space gamma-ray astrophysics: a Small Scientific Mission with optimized on-board and ground-segment resources.

The space program AGILE (Astro-rivelatore Gamma a Immagini LEggero) is a high-energy astrophysics mission supported by the Italian Space Agency (ASI) with scientific and programmatic participation by INAF, INFN, CNR, ENEA and several Italian universities (Tavani et al. 1998, 2000, 2002, 2008a; Barbiellini et al. 2000). The main industrial contractors include Carlo Gavazzi Space (CGS), Thales-AleniaSpace (formerly Laben), Rheinmetall Italia (formerly OerlikonContraves), Telespazio, Galileo Avionica, and Mipot.

The main scientific goal of the AGILE program is to provide a powerful and cost-effective mission with excellent imaging capability simultaneously in the $30 \mathrm{MeV}-50 \mathrm{GeV}$ and $18-60 \mathrm{keV}$ energy ranges with a large field of view that is unprecedented in high-energy astrophysics space missions.

AGILE was successfully launched by the Indian PSLVC8 rocket from the Sriharikota base on 2007 April 23 (see Fig. 1). The launch and orbital insertion were nominal, and a quasi-equatorial orbit was obtained with the smallest inclination ( 2.5 degrees) ever achieved by a high-energy space mission (e.g., SAS-2 and Beppo-SAX had inclinations of $\sim 4$ degrees). The satellite's commissioning phase was carried out during the period 2007 May-June. The scientific verification phase and the in-orbit calibration (based on long pointings at the Vela and Crab pulsars) were carried out during the period 2007 July-November. The nominal scientific observation phase (AGILE Cycle-1, AO1) started on 2007 December 1. AGILE is the first gamma-ray Mission successfully operating in space after the long temporal hiatus of almost ten years since the end of EGRET operations. It will operate in coexistence with the FERMI mission that was launched on 2008 June 11 (Michelson et al. 2008).

The AGILE instrument design is very innovative and based on solid-state Silicon detector technology and state-of-the-art

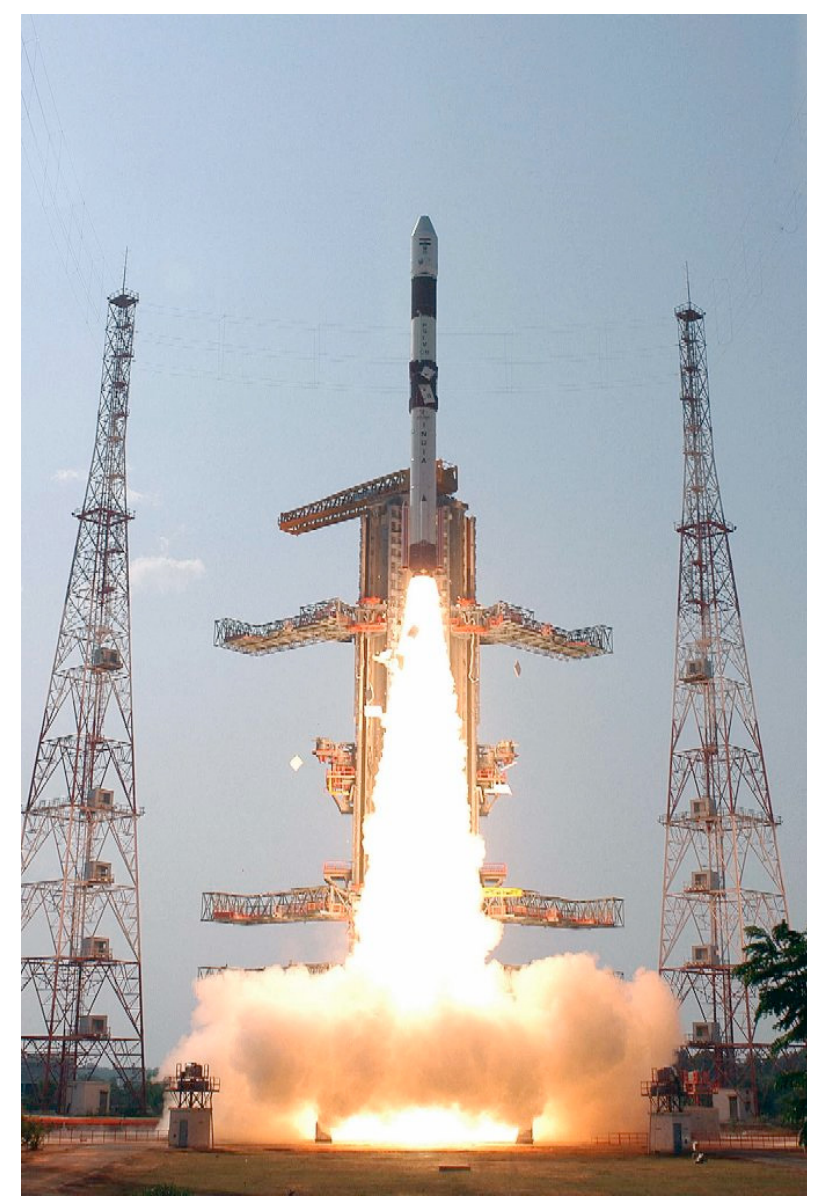

Fig. 1. The launch of the AGILE satellite by the Indian PSLV-C8 rocket from the Sriharikota ISRO base on 2007 April 23.

electronics and readout systems developed in Italian laboratories (Barbiellini et al. 1995a,b; Bakaldin et al. 1997). The instrument is light $(\sim 100 \mathrm{~kg})$ and very compact (see Fig. 3). The total satellite mass is about $350 \mathrm{~kg}$ (see Fig. 2).

AGILE is expected to substantially advance our knowledge in several research areas including the study of AGN and massive black holes, GRB, the unidentified gamma-ray sources, Galactic transient and steady compact objects, isolated and binary pulsars, pulsar wind nebulae (PWNae), supernova remnants, $\mathrm{TeV}$ sources, and the Galactic center, while mapping the overall gamma-ray emission from our Galaxy. Furthermore, the fast AGILE electronic readout and data processing (resulting in detector deadtimes less than $\sim 200 \mu$ s) allow a first systematic search for sub-millisecond gamma-ray/hard X-ray transients that are of interest for both Galactic compact objects (searching outburst durations comparable to the dynamical timescale of $\sim 1 M_{\odot}$ compact objects) and quantum gravity studies in extragalactic sources.

The AGILE Science Program is aimed at providing a complete sky coverage during its first year, while allowing a prompt response to gamma-ray transients and alert to follow-up multiwavelength observations. AGILE provides important information that is complementary to several high-energy space missions (CHANDRA, INTEGRAL, RXTE, XMM-NEWTON, SWIFT, SUSAXU, RHESSI, FERMI) and supports ground-based investigations in the radio, optical, and TeV bands. Part of the AGILE Science Program is open for guest investigations on a competitive basis. Quicklook data analysis and fast communication of 


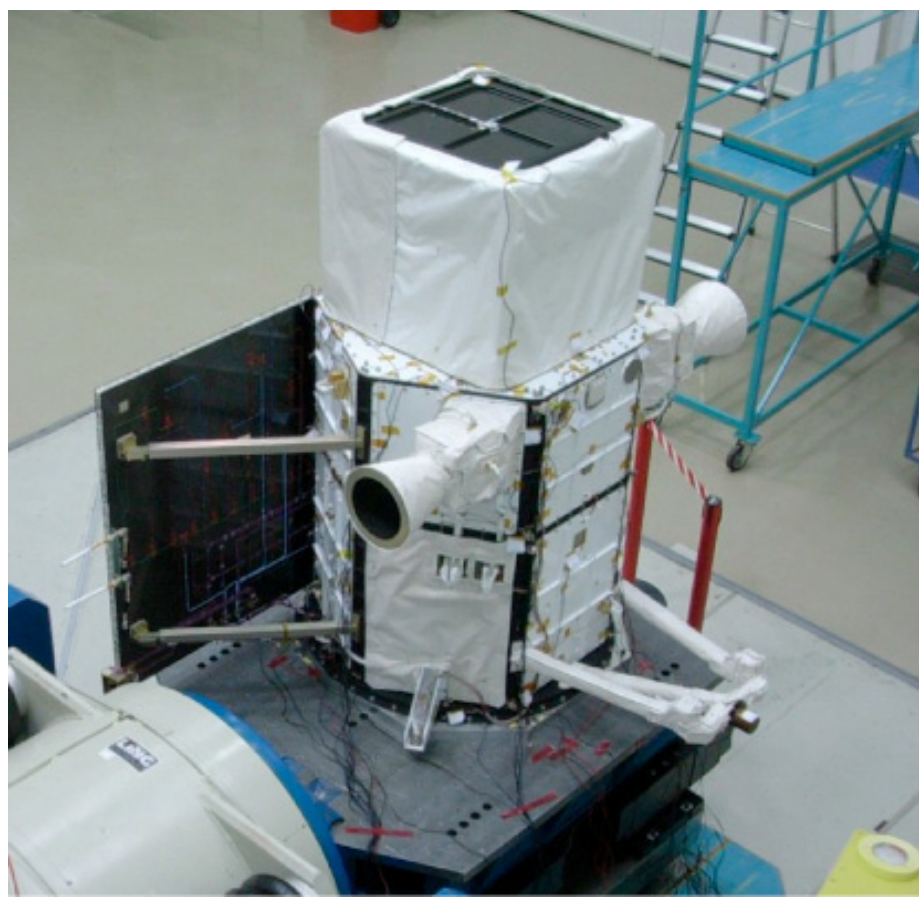

Fig. 2. The integrated AGILE satellite in its final configuration being covered by the thermal blanket during the qualification tests in IABG (Munich), July 2006. The total satellite mass is equal to $350 \mathrm{~kg}$.

new transients has been implemented as an essential part of the AGILE Science Program.

\section{Mission concept}

The AGILE program is motivated by very specific scientific requirements and goals. The essential point, permeating the whole mission from its conception, is to provide a very effective gamma-ray space instrument with excellent detection and imaging capabilities both in the gamma-ray and hard X-ray energy ranges. The very stringent mission constraints (satellite and instrument volume, weight, cost, and optimized ground segment) have determined a specific optimization strategy from the very beginning of the mission's development.

The AGILE mission challenge has been obtaining optimal gamma-ray/hard X-ray detection capabilitity (together with excellent timing resolution in the energy band near $1 \mathrm{MeV}$ ) with a very light $(\sim 100 \mathrm{~kg})$ instrument.

The AGILE instrument has therefore been designed and developed to obtain:

- excellent imaging capability in the energy range $100 \mathrm{MeV}-50 \mathrm{GeV}$, improving the EGRET angular resolution by a factor of 2 ;

- a very large field-of-view for both the gamma-ray imager (2.5 sr, i.e., 5 times larger than that of EGRET) and the hard X-ray imager (1 sr);

- excellent timing capability, with an overall photon absolute time-tagging uncertainty of $2 \mu \mathrm{s}$, coupled with very short deadtimes for gamma-ray detections;

- a good sensitivity to pointlike gamma-ray and hard X-ray sources. Depending on exposure and background, after a 1 -year program the flux sensitivity threshold can reach values of $(10-20) \times 10^{-8}$ photons $\mathrm{cm}^{-2} \mathrm{~s}^{-1}$ at energies above $100 \mathrm{MeV}$. The hard X-ray imager sensitivity is between 15 and $30 \mathrm{mCrab}$ at $20 \mathrm{keV}$ for a 1-day exposure over a $1 \mathrm{sr}$ field of view;

- good sensitivity to photons in the energy range $\sim$ 30-100 MeV, with an effective area above $200 \mathrm{~cm}^{2}$ at $30 \mathrm{MeV}$;

- a rapid response to gamma-ray transients and gammaray bursts, obtained by a special quicklook analysis program and coordinated ground-based and space observations; - accurate localization ( 2-3 arcmins) of GRBs and other transient events obtained by the GRID-SA combination (for typical hard X-ray transient fluxes above $\sim 1 \mathrm{Crab}$ ); the expected GRB detection rate for AGILE is $\sim 1-2$ per month;

- long-timescale continuous monitoring ( 2-3 weeks) of gamma-ray and hard X-ray sources;

- satellite repointing within $\sim 1$ day after special alerts.

The simultaneous hard X-ray and gamma-ray observations represent a novel approach to the study of high-energy sources. In the following, we address the main relevant features of the scientific performance of AGILE.

\section{Scientific objectives}

AGILE fits into the discovery path followed by previous gammaray missions (SAS-2, COS-B, and EGRET) and is complementary to FERMI. Nearly 270 gamma-ray sources above $30 \mathrm{MeV}$ were catalogued ${ }^{1}$ by EGRET (with only $30 \%$ identified as AGNs or isolated pulsars) (Hartman et al. 1999). We summarize here AGILE's main scientific objectives.

- Active galactic nuclei. For the first time, it is possible to monitor tens of potential gamma-ray emitting AGNs during each pointing. Several outstanding issues concerning the mechanism of AGN gamma-ray production and time evolution can be addressed by AGILE including: (1) the study of transient vs. low-level gamma-ray emission and duty cycles (Vercellone et al. 2004); (2) the relationship between the gamma-ray variability and the radio-optical-X-ray-TeV emission; (3) the possible correlation between relativistic radio plasmoid ejections and gamma-ray flares; (4) hard X-ray/gamma-ray correlations.

- Gamma-ray bursts. A few GRBs were detected by the EGRET spark chamber (Schneid et al. 1996). This number appeared to be limited by the EGRET FOV and sensitivity and not by the intrinsic GRB emission mechanism. Owing to a larger FOV, the GRB detection rate by the AGILE-GRID is expected to be higher than that of EGRET, i.e., 2-5 events/year. Furthermore, the short GRID deadtime ( $\sim 500$ times shorter than that of EGRET) allows for a better study of the initial phase of GRB pulses (for which EGRET response was in some cases inadequate). The hard X-ray imager (Super-AGILE) can localize GRBs within a few arcminutes and can systematically study the interplay between hard X-ray and gamma-ray emissions. Special emphasis is given to the search for sub-millisecond GRB pulses independently detectable by the Si-tracker, MCAL, and SuperAGILE.

- Diffuse Galactic gamma-ray emission. The AGILE good angular resolution and large average exposure further improves our knowledge of cosmic ray origin, propagation, interaction, and emission processes. A detailed gamma-ray imaging of individual molecular cloud complexes is possible.

- Gamma-ray pulsars and PWNae. AGILE will contribute to the study of gamma-ray pulsars (PSRs) in several ways: (1) improving timing and lightcurves of known gamma-ray PSRs,

\footnotetext{
1 See also the analysis of Casandjian \& Grenier (2008).
} 
(2) improving photon statistics for blind gamma-ray period searches of pulsar candidates, (3) studying unpulsed gammaray emission from plerions in supernova remnants and studying pulsar wind/nebula interactions, e.g., as in the Galactic sources recently discovered in the $\mathrm{TeV}$ range (Aharonian et al. 2006). Particularly interesting for AGILE are the $\sim 30$ new young PSRs discovered in the Galactic plane by the Parkes survey (Kramer et al. 2003).

- The search for non-blazar gamma-ray variable sources in the Galactic plane, currently a new class of unidentified gamma-ray sources such as GRO J1838-04 (Tavani et al. 1997).

- Compact Galactic sources, micro-quasars, new transients. A large number of gamma-ray sources near the Galactic plane are unidentified, and sources such as 2CG 135+1/LS I+61 303 can be monitored on timescales of months. Cyg X-1 is also monitored, and gamma-ray emission above $30 \mathrm{MeV}$ will be intensively searched. Galactic X-ray jet sources (such as Cyg X-3, GRS 1915+105, GRO J1655-40 and others) can produce detectable gamma-ray emission for favorable jet geometries, and a TOO program is planned to follow-up new discoveries of micro-quasars.

- Supernova remnants (SNRs). Several possible gammaray source-SNR associations were proposed based on EGRET data (Sturner \& Dermer 1995; Esposito et al. 1996). However, none were decisive. High-resolution imaging of SNRs in the gamma-ray range can provide the missing information to decide between leptonic and hadronic models of SNR emission above $100 \mathrm{MeV}$.

- Fundamental physics: quantum gravity. AGILE detectors are suited for quantum gravity (QG) studies. The existence of sub-millisecond GRB pulses lasting hundreds of microseconds (Bhat et al. 1992) opens the way to studying QG delay propagation effects by AGILE detectors. Particularly important is the AGILE mini-calorimeter trigger logic with photon-by-photon independent readout for each of the $30 \mathrm{CsI}$ bars of short deadtime $(\sim 20 \mu \mathrm{s})$ and absolute timing resolution $(\sim 3 \mu \mathrm{s})$. Energy-dependent time delays near $\sim 100 \mu$ s for ultrashort GRB pulses in the energy range $0.3-3 \mathrm{MeV}$ can be detected. If these GRB ultra-short pulses originate at cosmological distances, sensitivity to the Planck's mass can be reached by AGILE.

\section{Main characteristics of the mission}

In order to make substantial progress with respect to the previous generation of gamma-ray astrophysics missions and to substantially contribute to the current and future observations, the AGILE instrument is required to achieve an optimal performance with the following characteristics.

\subsection{Gamma-ray and X-ray angular resolution}

The gamma-ray detection resolution is required to achieve an effective PSF with $68 \%$ containment radius of $1^{\circ}-2^{\circ}$ at $E>$ $300 \mathrm{MeV}$ allowing a gamma-ray source positioning with error box radii in the range $6^{\prime}-20^{\prime}$ depending on source spectrum, intensity, and sky position. The hard X-ray imager operating in the $18-60 \mathrm{keV}$ band has a spatial resolution of 3 arcmin (pixel size). This translates into a pointing reconstruction of 1-2 arcmins for relatively strong transients at the Crab flux level.

\subsection{Large FOVs of the gamma-ray and hard X-ray imagers}

A crucial feature of the AGILE instrument is its very large field of view for both the gamma-ray and hard X-ray detectors. The gamma-ray FOV is required to be $2.5-3$ sr, i.e., to cover about $1 / 5$ of the entire sky. The hard X-ray imager is required to cover a region of about $1 \mathrm{sr}$. The combination of coaligned gamma-ray and hard X-ray imagers with very large FOVs is unprecedented and is the main novelty of the AGILE instrument configuration.

\subsection{Fast reaction to strong high-energy transients}

The existence of a large number of variable gamma-ray sources, e.g., near the Galactic plane Tavani et al. (1997) requires a reliable program for quick response to transient gamma-ray emission. Quicklook Analysis of gamma-ray data is an important task of the AGILE Mission. Prompt communication of bright gamma-ray transients (above $10^{-6} \mathrm{ph} \mathrm{cm}^{-2} \mathrm{~s}^{-1}$, requiring typically 1-2 days to be detected with high confidence) is ensured by a proper ground segment configuration. Alerts for shorter timescale (seconds/minutes/hours) transients (GRBs, SGRs, and other bursting events) are also possible. A primary responsibility of the AGILE Team is to provide positioning of short-timescale transient that is as accurate as possible and to alert the community though dedicated channels.

\subsection{A large exposure for Galactic and extragalactic sources}

Owing to a larger effective area at large off-axis angles, the AGILE average exposure above $100 \mathrm{MeV}$ typically will be 4 times greater than that of EGRET after a 1-year sky survey. Deep exposures for selected regions of the sky can be obtained with repeated overlapping pointings. This can be particularly useful to monitor simultaneously selected Galactic and extragalactic sources. The typical AGILE 1-day exposure at $100 \mathrm{MeV}$ for a source in the central part of the FOV is $\sim 10^{7} \mathrm{~cm}^{2} \mathrm{~s}^{-1}$ that turns out to be comparable with that of FERMI in the same energy and time range.

\subsection{High-precision timing}

AGILE detectors have optimal timing capabilities. The on-board navigation system makes possible an absolute time tagging precision of $2 \mu \mathrm{s}$ for individual photons. Depending on the event characteristics, absolute time tagging can achieve values near $2 \mu \mathrm{s}$ for the Silicon-tracker, and 3-4 $\mu$ s for the mini-calorimeter and Super-AGILE.

Also the instrumental AGILE deadtime is very small for gamma-ray detection (typically less than $200 \mu \mathrm{s}$ ). After taking the segmentation of the electronic readout of MCAL and Super-AGILE detectors (30 MCAL elements and 16 SuperAGILE elements) into account, the MCAL and SA effective deadtimes are substantially less than for the individual units. We reach $\sim 2 \mu$ s for MCAL, and $5 \mu$ s for SA. Furthermore, a special memory ensures that MCAL events detected during the Si-tracker readout deadtime are automatically stored in the GRID event. For these events, precise timing and detection in the $\sim 1-200 \mathrm{MeV}$ range can be achieved with temporal resolution well below $100 \mu$ s. This may be very relevant for AGILE high-precision timing investigations. 


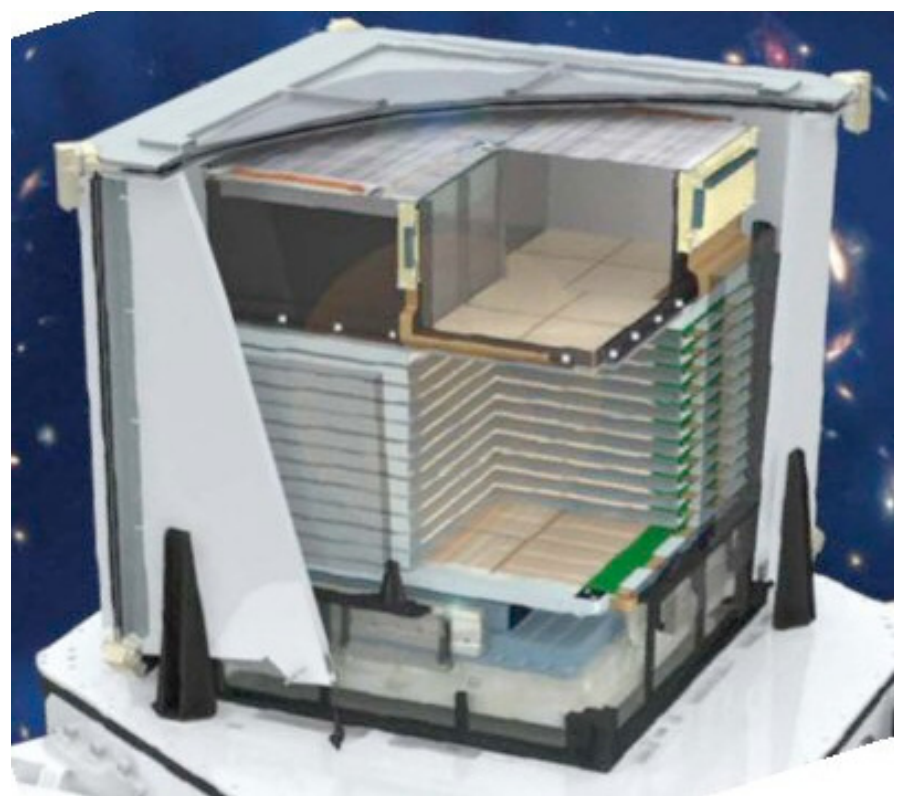

Fig. 3. The AGILE scientific instrument showing the hard X-ray imager, the gamma-ray tracker, and calorimeter. The anticoincidence system is partially displayed, and no lateral electronic boards and harness are shown for simplicity. The AGILE instrument "core" is approximately a cube of about $60 \mathrm{~cm}$ size and of weight approximately equal to $100 \mathrm{~kg}$.

\section{The scientific instrument}

The AGILE scientific payload is made of three detectors combined into one instrument with broad-band detection and imaging capabilities. The payload is surrounded by an anticoincidence system to screen out the charged particle background. A sophisticated data handling system completes the instrument. Figure 3 shows a schematic view of the instrument, and Table 1 summarizes the main instrument's scientific performance. We summarize here the main characteristics of the instrument: several papers describe the individual detectors in detail (Perotti et al. 2006; Barbiellini et al. 1995a; Prest et al. 2003; Feroci et al. 2007; Labanti et al. 2006).

The Gamma-Ray Imaging Detector (GRID) is sensitive in the energy range $\sim 30 \mathrm{MeV}-50 \mathrm{GeV}$, and consists of a silicontungsten tracker, a Cesium Iodide calorimeter, and an anticoincidence system.

The GRID trigger logic and data acquisition system (based on anticoincidence, tracker and mini-calorimeter information) allows for an efficient background discrimination and inclined photon acceptance (Tavani et al. 2008b; Argan et al. 2008b). The GRID is designed to achieve an optimal angular resolution (source location accuracy $\sim 6^{\prime}-12^{\prime}$ for intense sources), a very large field-of-view ( $\sim 2.5 \mathrm{sr})$, and a sensitivity comparable to that of EGRET for sources within 10-20 degree from the main axis direction (and substantially better for larger off-axis angles).

The hard X-ray Imager (Super-AGILE) is a unique feature of the AGILE instrument (for a complete description, see Feroci et al. 2007). The imager is placed on top of the gammaray detector and is sensitive in the $18-60 \mathrm{keV}$ band.

A mini-calorimeter operating in the "burst mode" is the third AGILE detector. It is part of the GRID, but also also capable of independently detecting GRBs and other transients in the $350 \mathrm{keV}-100 \mathrm{MeV}$ energy range with excellent timing capabilities.

Figure 2 shows the integrated AGILE satellite and Fig. 3 a schematic representation of the instrument. We briefly describe here the main detecting units of the AGILE instrument, more detailed information will be presented elsewhere.

\subsection{The anticoincidence system}

The anticoincidence (AC) system is aimed at a very efficient charged particle background rejection (Perotti et al. 2006). It also allows a preliminary direction reconstruction for triggered photon events through the DH logic. The AC system completely surrounds all AGILE detectors (Super-AGILE, Si-tracker and MCAL). Each lateral face is segmented in three plastic scintillator layers $(0.6 \mathrm{~cm}$ thick) connected to photomultipliers placed at the bottom of the panels. A single plastic scintillator layer $(0.5 \mathrm{~cm}$ thick) constitutes the top-AC whose signal is read by four light photomultipliers placed at the four corners of the structure frame. The segmentation of the AC System and the Silicon tracker trigger logic contribute in an essential way to produce the very large field of view of the AGILE-GRID.

\subsection{The Silicon-tracker}

The Silicon tracker (ST) is the AGILE gamma-ray imager based on photon conversion into electron-positron pairs (Barbiellini et al. 1995b; Prest et al. 2003). It consists of a total of 12 trays with a repetition pattern of $1.9 \mathrm{~cm}$ (Fig. 4). The first 10 trays are capable of converting gamma-rays by a Tungsten layer. Tracking of charged particles is ensured by silicon microstrip detectors that are configured to provide the two orthogonal coordinates for each element (point) along the track. The individual silicon detector element is a tile of area $9.5 \times 9.5 \mathrm{~cm}^{2}$, microstrip pitch of $121 \mu \mathrm{m}$, and $410 \mu \mathrm{m}$ thickness. Four silicon tiles are bonded together to provide a ladder. Four ladders constitute a ST plane. The AGILE ST readout system is capable of detecting and storing the energy deposited in the silicon microstrips by the penetrating particles. The readout signal is processed for half of the microstrips by an alternating readout system characterized by "readout" and "floating strips". The analog signal produced in the readout strips is read and stored for further processing. Each silicon ladder has a total of 384 readout channels $(242 \mu \mathrm{m}$ readout pitch) and 3 TAA1 chips are required to process the analog signal from the readout strips independently. Each Si-tracker layer is then made of $4 \times 4$ Si-tiles, for a total geometric area of $38 \times 38 \mathrm{~cm}^{2}$. The first 10 trays are equipped with a Tungsten layer of $245 \mu \mathrm{m}\left(0.07 X_{0}\right)$ positioned in the bottom part of the tray. The two orthogonal coordinates of particle hits in the ST are provided by two layers of Silicon detectors properly configured for each tray that therefore has $2 \times 1,536$ readout microstrips. Since the ST trigger requires a signal from at least three (contiguous) planes, two more trays are inserted at the bottom of the tracker without the Tungsten layers. The total readout channel number for the GRID tracker is then 36,864 . The $1.9 \mathrm{~cm}$ distance between mid-planes has been optimized through extensive Montecarlo simulations. The ST has an onaxis total radiation length near $0.8 X_{0}$. Special trigger logic algorithms implemented on-board (Level-1 and Level-2) lead to a substantial particle/albedo-photon background subtraction and a preliminary on-board reconstruction of the photon incidence angle. Both digital and analog information are crucial for this task. Figure 5 shows a typical read-out configuration of a gamma-ray event detected by the AGILE Silicon tracker. The positional resolution obtained by the ST is excellent, being below $40 \mu \mathrm{m}$ for a wide range of particle incidence angles Barbiellini et al. (2002). 
Table 1. The AGILE cientific Performance.

\begin{tabular}{lc}
\hline \hline Gamma-ray imaging detector (GRID) & \\
\hline Energy range & $30 \mathrm{MeV}-50 \mathrm{GeV}$ \\
Field of view & $\sim 2.5 \mathrm{sr}$ \\
Flux sensitivity $\left(E>100 \mathrm{MeV}, 5 \sigma\right.$ in $\left.10^{6} \mathrm{~s}\right)$ & $3 \times 10^{-7}\left(\mathrm{ph} \mathrm{cm}^{-2} \mathrm{~s}^{-1}\right)$ \\
Angular resolution at $100 \mathrm{MeV}(68 \%$ cont. radius) & 3.5 degrees \\
Angular resolution at $400 \mathrm{MeV}(68 \%$ cont. radius) & 1.2 degrees \\
Source location accuracy (high Gal. lat., 90\% C.L.) & $\sim 15$ arcmin \\
Energy resolution (at 400 MeV) & $\Delta E / E \sim 1$ \\
Absolute time resolution & $\sim 2 \mu \mathrm{s}$ \\
Deadtime & $\sim 100-200 \mu \mathrm{s}$ \\
\hline Hard X-ray imaging detector (Super-AGILE) & $18-60 \mathrm{keV}$ \\
Energy range & $107^{\circ} \times 68^{\circ}$ \\
Single (1-dim.) detector FOV (FW at zero sens.) & $68^{\circ} \times 68^{\circ}$ \\
Combined (2-dim.) detector FOV (FW at zero sens.) & $\sim 15 \mathrm{mCrab}$ \\
Sensitivity (18-60 keV, 5 $\sigma$ in 1 day) & $6 \mathrm{arcmin}$ \\
Angular resolution (pixel size) & $\sim 1-2 \mathrm{arcmin}$ \\
Source location accuracy $(S / N \sim 10)$ & $\Delta E \sim 8 \mathrm{keV}$ \\
Energy resolution (FWHM) & $\sim 2 \mu \mathrm{s}$ \\
Absolute time resolution & \\
\hline Mini-calorimeter & $0.35-50 \mathrm{MeV}$ \\
Energy range & $13 \% \mathrm{FWHM}$ \\
Energy resolution ( at $1.3 \mathrm{MeV}$ ) & $\sim 3 \mu \mathrm{s}$ \\
Absolute time resolution & $\sim 20 \mu \mathrm{s}$ \\
Deadtime (for each of the 30 CsI bars) & \\
\hline
\end{tabular}

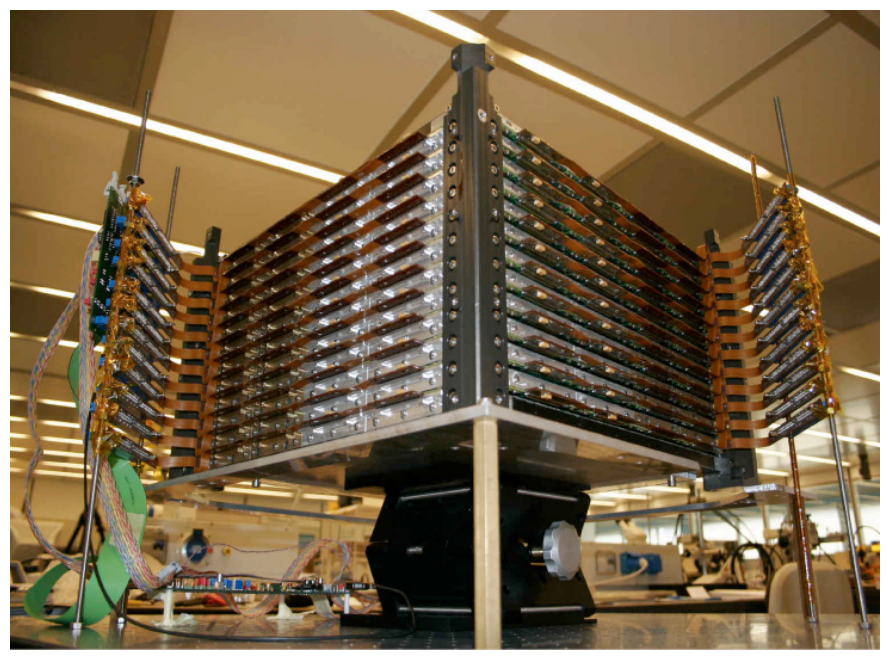

Fig. 4. The assembled AGILE Silicon tracker developed in the Trieste INFN laboratories and in the Mipot facility in Cormons (Italy) before being integrated with the rest of the instrument (June 2005).

\subsection{Super-AGILE}

Super-AGILE (SA), the ultra-compact and light hard-X-ray imager of AGILE (Feroci et al. 2007) is a coded-mask system made of a silicon detector plane and a thin tungsten mask positioned $14 \mathrm{~cm}$ above it (Fig. 6). The detector plane is organized in four independent, square silicon detectors $\left(19 \times 19 \mathrm{~cm}^{2}\right.$ each) plus dedicated front-end electronics based on the XAA1.2 chips suitable to the SA energy range Del Monte et al. (2007). The total number of SA readout channels is 6,144 . The detection cabability of SA includes: (1) photon-by-photon transmission and imaging of sources in the energy range $18-60 \mathrm{keV}$, with a large field-of-view (FOV $\sim 1 \mathrm{sr}$ ); (2) an angular resolution of 6 arcmin; (3) good sensitivity ( 15 mCrab between $18-60 \mathrm{keV}$ for $50 \mathrm{ks}$ integration, and $\lesssim 1 \mathrm{Crab}$ for a few seconds integration). SA is aimed at the hard X-ray detection simultaneously with gamma-ray detection of high-energy sources with excellent
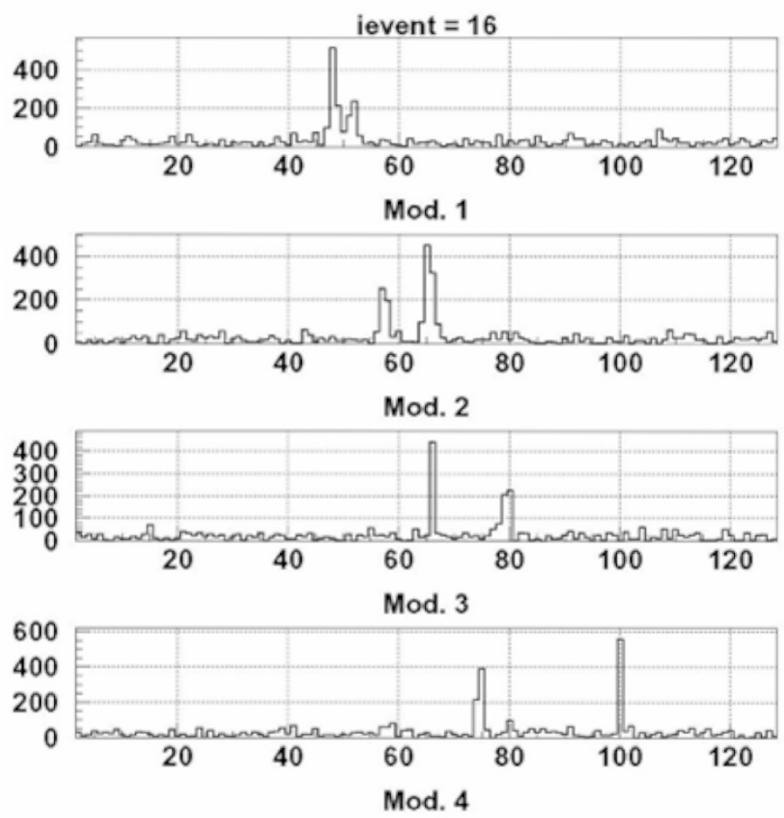

Fig. 5. Detection of a typical gamma-ray event by the AGILE tracker in the Thales-Alenia Space laboratory in Milan during integration tests (July 2005). The electron-positron hits in the Silicon microstrips produce typical "clusters" of read-out strips whose analog signals produce a sequence of deposited energy "histograms". This cluster positioning capability is a unique feature of the AGILE gamma-ray imager.

timing capabilities (a few microseconds). The SA acquisition logic produces essential GRB quantities on-board such as time, coordinates, and preliminary flux estimates. The AGILE satellite is equipped with an ORBCOMM transponder capable of trasmitting the GRB on-board processed physical quantities to the ground within $10-30 \mathrm{mn}$. 


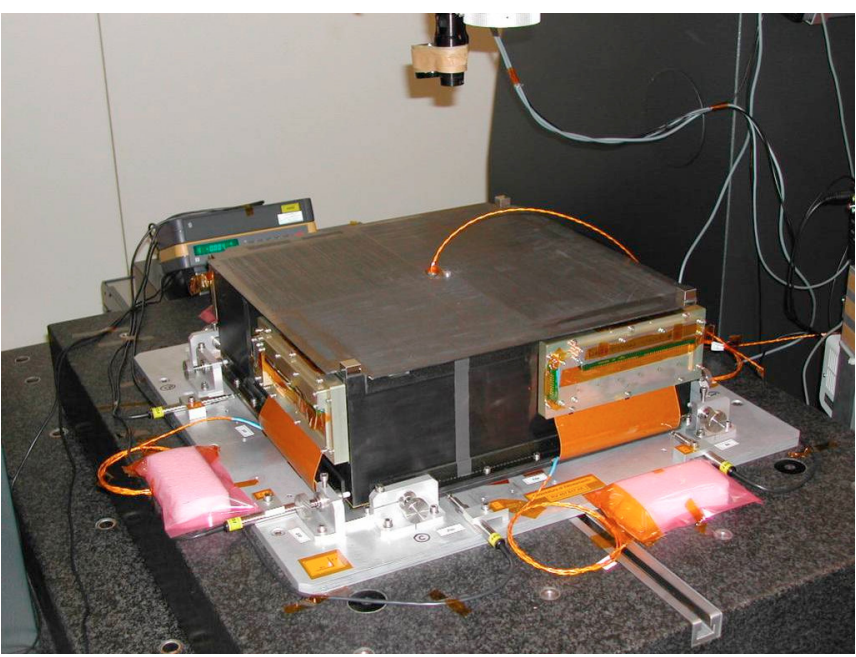

Fig. 6. The Super-AGILE detector in the INAF-IASF-Rome laboratory during metrology measurements (March 2005).

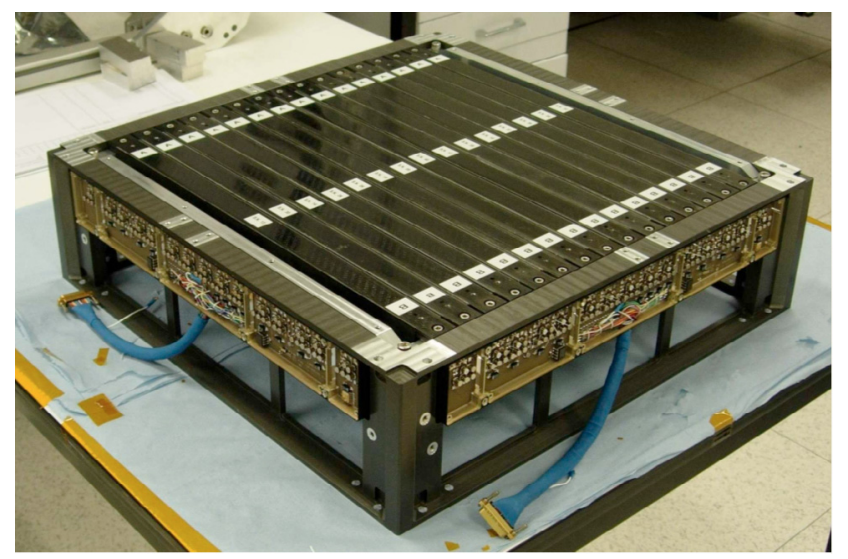

Fig. 7. The MCAL detector consisting of two planes of CsI bars enclosed in carbon fiber supporting structure is shown during the integration process with the signal readout diodes and FFE in the ThalesAlenia Space laboratories in Milan (February, 2005).

\subsection{The mini-calorimeter}

The mini-calorimeter (MCAL) is made of 30 Caesium Iodide $(\mathrm{CsI}(\mathrm{Tl}))$ bars arranged in two planes, for a total (on-axis) radiation length of $1.5 X_{0}$ (see Fig. 7). A detailed description of the MCAL detector can be found in Labanti et al. (2006, 2008). The signal from each CsI bar is collected by two photodiodes placed at both ends. The MCAL aims are: (i) obtaining information on the energy deposited in the CsI bars by particles produced in the Silicon tracker (and therefore contributing to the determination of the total photon energy); (ii) detecting GRBs and other impulsive events with spectral and intensity information in the energy band $\sim 0.35-100 \mathrm{MeV}$. An independent burst search algorithm is implemented on board with a wide range of trigger timescales for an MCAL independent GRB detection. Following a GRB trigger, MCAL is indeed able to store photon-by-photon information for a duration dynamically determined by the onboard logic. The MCAL segmentation and the photon-particle hit positioning along the bars allow one to obtain the general configuration of "hits" across the calorimeter volume. This information is used by the on-board trigger logic for background discrimination and by the ground processing to obtain a preliminary determination of GRB direction.

\subsection{Data handling and power supply}

The Data Handling (DH) and power supply systems complete the instrument. The DH is optimized for fast on-board processing of the GRID, MCAL and Super-AGILE data (Argan et al. 2004; Tavani et al. 2008b; Argan et al. 2008b). Given the relatively large number of readable channels in the ST and SuperAGILE ( 40 000), the instrument requires a very efficient onboard data processing system. The GRID trigger logic for the acquisition of gamma-ray photon data and background rejection is structured in two main levels: Level-1 and Level-2 trigger stages. The Level-1 trigger is fast $(\lesssim 5 \mu \mathrm{s})$ and requires a signal in at least three out of four contiguous tracker planes, and a proper combination of fired TAA1 chip number and AC signals. An intermediate Level-1.5 stage is also envisioned (lasting $\sim 20 \mu \mathrm{s}$ ), with the acquisition of the event topology based on the identification of fired TAA1 chips. Both Level-1 and Level-1.5 have a hardware-oriented veto logic providing a first cut of background events. Level-2 data processing includes a GRID readout and pre-processing, "cluster data acquisition" (analog and digital information). The Level-2 processing is asynchronous (estimated duration $\sim$ a few $\mathrm{ms}$ ) with the actual GRID event processing. The GRID deadtime turns out to be $\lesssim 200 \mu$ s and is dominated by the tracker readout.

The charged particle and albedo-photon background passing the Level-1+1.5 trigger level of processing is measured in orbit to be $\lesssim 100$ events/sec for the equatorial orbit of AGILE. The on-board Level-2 processing has the task of reducing this background by a factor between 3 and 5. Off-line ground processing of the GRID data has the goal to reduce the particle and albedophoton background rate above $100 \mathrm{MeV}$ to the expected rate of $\sim 0.02$ events/sec.

To maximize the GRID FOV and detection efficiency for large-angle incident gamma-rays (and minimize the effects of particle backsplash from the MCAL and of "Earth albedo" background photons), the data acquisition logic uses proper combinations of top and lateral AC signals and a coarse on-line direction reconstruction in the ST. For laterally incident events depositing more than $200 \mathrm{MeV}$ in the MCAL, the AC veto may be disabled to allow the acquisition of gamma-ray photon events with energies greater than $1 \mathrm{GeV}$.

A special set of memory buffers and burst search algorithms are implemented to maximize data acquisition for transient gamma-ray events (e.g., GRBs) in the ST, Super-AGILE and MCAL, respectively. The Super-AGILE event acquisition envisions a first "filtering" based on AC-veto signals, and pulseheight discrimination in the front-end electronics. The events are then buffered and transmitted to the CPU for burst searching and final data formatting. The four Si-detectors of Super-AGILE are organized in sixteen independent readout units, resulting in a $\sim 5 \mu$ s global deadtime.

To maximize the detecting area and minimize the instrument weight, the GRID and Super-AGILE front-end-electronics are partly accommodated in special boards placed externally on the tracker lateral sides. Electronic boxes and P/L memory (and buffer) units are positioned at the bottom of the instrument within the spacecraft.

\subsection{The AGILE instrument calibration}

The AGILE scientific instrument was fully calibrated on the ground during a set of calibration campaigns dedicated to the three instrument detectors. We briefly summarize here the main 
calibration operations, postponing a detailed analysis of the campaigns to forthcoming papers.

\subsubsection{The gamma-ray imager calibration}

The AGILE-GRID calibration was carried out at the INFN National Laboratories in Frascati (LNF) during the period 2005 November 1-25.

A beam of gamma-ray photons in the energy range 20-700 MeV was produced by electron Bremsstrahlung in the LNL Beam Test Facility. The produced Gamma-rays were timetagged by a dedicated system based on the measurement by silicon strip detectors of the electrons' trajectories in a bending magnetic field. A total of 100000 photon tagged events was accumulated for several incidence directions and instrument configurations. Both the GRID spectral and PSF response were carefully studied and compared with results of extensive simulations (Pucella et al. 2008; Longo et al. 2008). Furthermore, the leptonic background was studied by using the direct electron and positron beams interacting with the AGILE GRID for different geometries. A sequence of runs was obtained for both direct incidence on the instrument, as well as for events originating by interactions with the spacecraft.

\subsubsection{The hard X-ray imager calibration}

The Super-AGILE imager was calibrated at different stages during the instrument integration and testing. It was first calibrated at the detection plane and stand-alone detector level in the clean room of INAF-IASF Rome on 2005 April and August, respectively. The SA effective area and intrinsic imaging properties were investigated by means of a highly collimated X-ray tube and point-like radioactive sources (see Donnarumma et al. 2006; Evangelista et al. 2006, for details). A dedicated procedure was developed (Donnarumma et al. 2008) to correct the SA images for the beam divergence in order to derive imaging calibration properties from measurements with radioactive sources at finite distance (about $200 \mathrm{~cm}$ from the experiment).

Once integrated with the instrument and satellite, the SA imager was then fully calibrated in 2007 January at the CGS facility in Tortona. A sequence of measurements were carried out with radioactive sources positioned at different angles with respect to the instrument axis. The imaging response was studied as a function of the source position in the field of view (in more than 40 positions) and energy (at 22, 30 and $60 \mathrm{keV}$ ). Radioactive sources were held at a $200 \mathrm{~cm}$ distance and their position were independently measured by Super-AGILE and by an optical laser tracking system (in collaboration with the metrology group of ENEA Frascati). The calibration campaign envisaged a total of more than 110 measurements and $340 \mathrm{ks}$ livetime, with more than $10^{7}$ source photons collected. The data analysis allowed us to calibrate the imaging and spectral response of SA. Figure 8 shows a sample of the results achieved during the final ground calibrations, confirming the expected $6 \operatorname{arcmin}(F W H M)$ point spread function (PSF) and the $\sim 1-2$ arcmin point source location accuracy (see Feroci et al. 2007 and Evangelista et al. 2008 , for more details). SA on-board imaging was also tested in Tortona. A sequence of GRB simulating tests were carried out to check the on-board trigger logic and parameter setting.

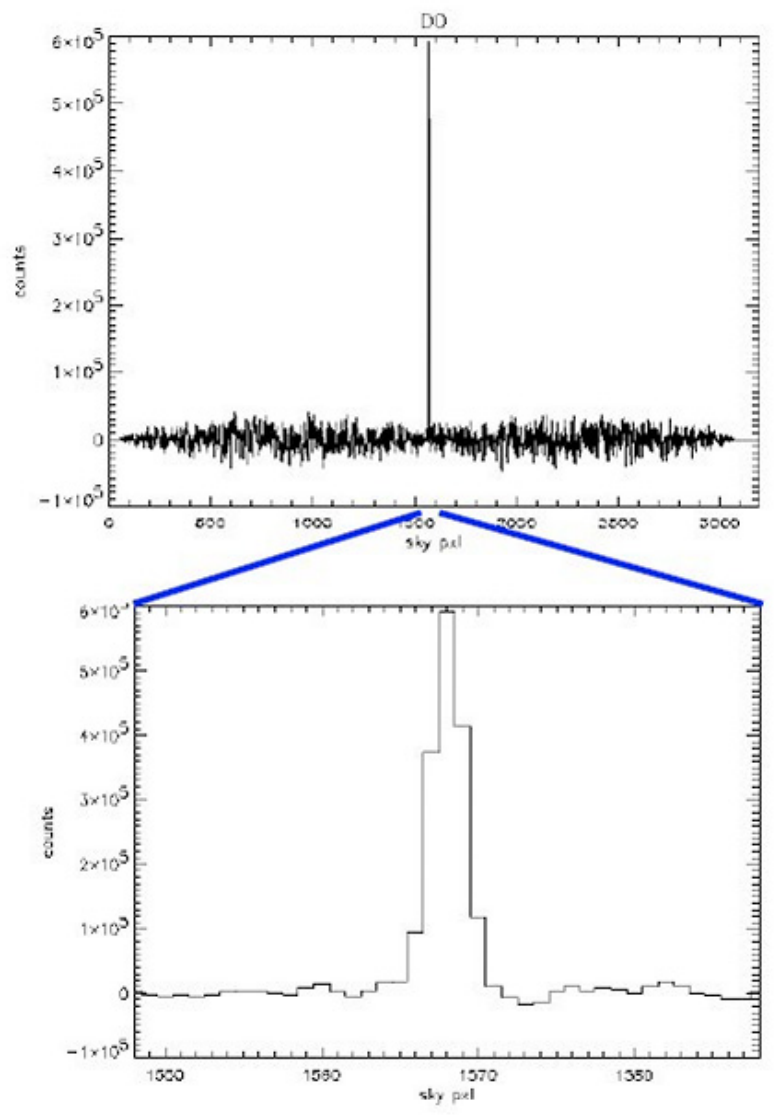

Fig. 8. Calibration data of the Super-AGILE imaging detector obtained with a $22 \mathrm{keV}$ radioactive source placed at a $200 \mathrm{~cm}$ distance, after correcting for beam divergence effects.

\subsubsection{The calorimeter calibration}

Several calibration sessions of MCAL were carried out after its integration (Labanti et al. 2008). A first stand-alone calibration session was performed at instrument level, prior to integration into the AGILE payload, using a collimated ${ }^{22} \mathrm{Na}$ radioactive source. By these measurements the bars physical parameters, such as the light output and the light attenuation coefficients, were obtained. After the instrument integration, MCAL was tested at the DAФNE accelerator Beam Test Facility in Frascati during the GRID calibration session. MCAL was then calibrated after satellite integration at the CGS facility in Tortona, exposing the instrument to an uncollimated ${ }^{22} \mathrm{Na}$ radioactive source placed at different positions with respect to the satellite axis in order to evaluate the MCAL efficiency and the overall contribution of the spacecraft volumes to the detector response.

The MCAL response to impulsive events and GRBs was studied by reproducing the conditions for GRB events of durations between $16 \mathrm{~ms}$ and $2 \mathrm{~s}$ by means of a dedicated setup mainly based on moving a radioactive source behind a collimator; the speed of the source determining the duration and rise time of the Burst (Fuschino et al. 2008). With this setup all time windows of the burst search logic above $16 \mathrm{~ms}$ have been stimulated and tested.

\subsubsection{The anticoincidence system calibration}

An excellent anticoincidence system is required for an efficient background rejection of the AGILE instrument. The AC flight 


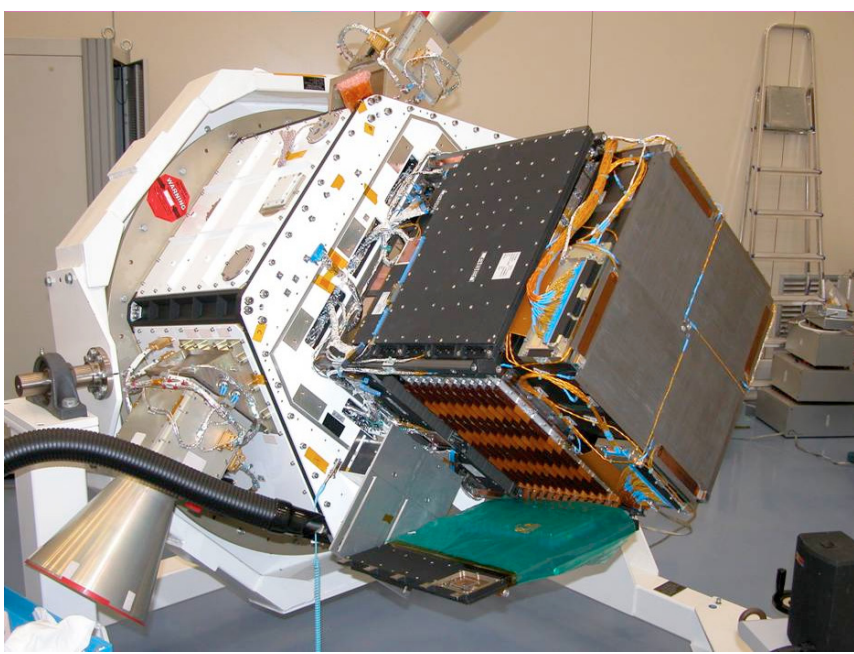

Fig. 9. The AGILE "Payload Unit" consisting of the integrated instrument (here shown without the anticoincidence system during integration tests) and the upper part of the satellite (November 2006).

units (scintillator panels and photo-multiplier assemblies) have been extensively calibrated in a dedicated run at the CERN PS T9 beam line facility during the month of August 2004 (see Perotti et al. 2006, for a detailed presentation). The particle detection inefficiency of the top panel and lateral panels plus their analog FEE was measured to be better than $6 \times 10^{-6}$ and $4 \times 10^{-5}$, respectively. Adding the effect of the FEE discrimination system somewhat increases the inefficiencies. The final result is that the AGILE AC inefficiencies are measured to be below the required value of $10^{-4}$ for all panels.

\section{The AGILE satellite}

The AGILE spacecraft is of the MITA class and has been developed by Carlo Gavazzi Space (CGS) as prime contractor and by Rheinmetall Italia (formerly Oerlikon-Contraves). The spacecraft has been customized for the AGILE program, and represents a very good compromise between the mission technical requirements and the stringent volume and weight constraints.

The overall configuration has an hexagonal shape (for volume optimization and heat dissipation requirements) and is divided into two units: (1) the "Payload Unit" (see Fig. 9) hosting the instrument, its electronics, the instrument DH computer unit, the star sensors and electronics, the navigation unit and electronics; (2) a "service module" hosting all the other vital control units and the satellite OBDH system. The instrument is mechanically and electrically integrated with the upper unit.

The AGILE spacecraft provides all the required services for the satellite operations and the AGILE instrument optimal functioning. In particular, it provides the power supply, communication with the ground station, satellite attitude control and thermal control capability. All these functionalities are managed by the On Board Data Handling (OBDH) subsystem that also takes care of the on-board monitoring and control activities.

To guarantee the required reliability for the whole mission lifetime, all main spacecraft units have a full redundancy. The redundancy management is mainly performed autonomously by the OBDH software in order to provide the required autonomy to recover from possible subsystem failures that can occur during the mission.

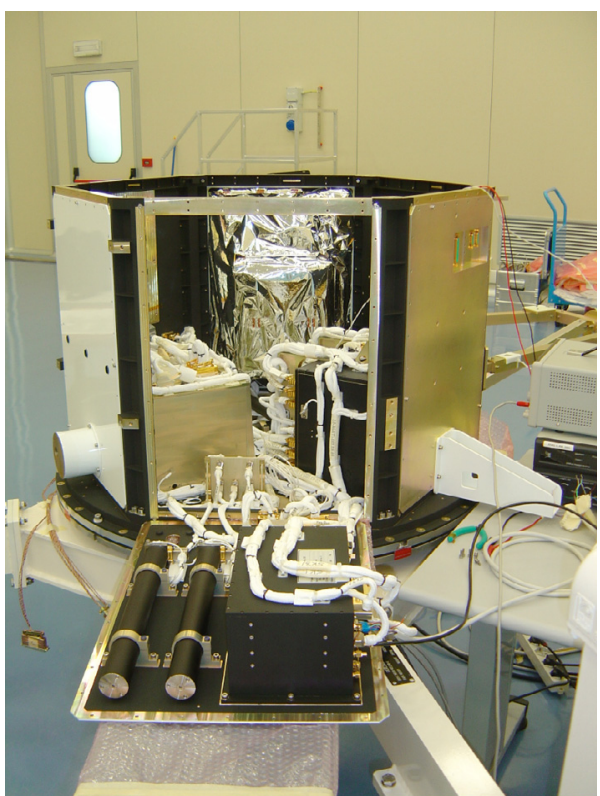

Fig. 10. The AGILE platform during the integration tests in Tortona (Italy).

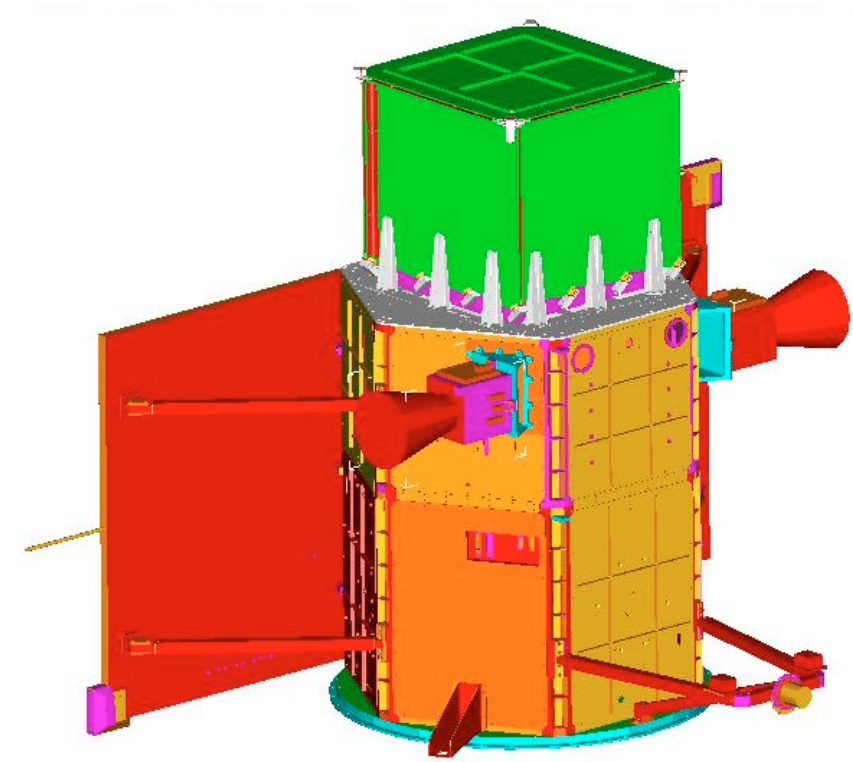

Fig. 11. A schematic view of the AGILE satellite configuration.

The satellite thermal control is of passive type and is implemented by placing the most dissipating devices close to the externally radiating surfaces. A set of heaters, controlled by thermostats or by software are used on critical equipments (in particular the Li-Ion battery and sensitive parts of the instrument such as the AC system and the MCAL) to keep the temperatures within the required range.

\subsection{Solar panels and power system}

The satellite electrical power is provided by a fixed solar panel of about $2 \mathrm{~m}^{2}$ area, equipped with high-efficiency triple junction GaAs cells. The solar panel system is positioned along the satellite body in order to minimize the secondary particle 
background for the instrument. To supply power during the eclipse periods and during the attitude acquisition phase, the power system includes a re-chargeable lithium-ion battery with a capacity of 33 Ah. The battery-charging activity, the power conversion, and distribution to the satellite subsystems is provided by a power unit controlled by the $\mathrm{OBDH}$ through a dedicated software.

The fixed solar panels configuration constrains the AGILE pointing strategy. The Sun direction is required to be always quasi-orthogonal (within 1-3 degrees) to the solar panel surface. This implies that AGILE cannot point towards the Sun: most of the sky is accessible by the large FOV AGILE gamma-ray imager but the solar and anti-solar directions are excluded from direct pointings.

\subsection{Satellite attitude control}

The AGILE satellite attitude control system (ACS) is designed to achieve the scientific requirement of the satellite attitude reconstruction of $\sim 1$ arcmin. The AGILE ACS uses a set of sensors and actuators controlled by a dedicated software running on the $\mathrm{OBDH}$ computer to guarantee the required attitude pointing in all the mission phases. After the launcher separation, the ACS was able to acquire, within a few orbits, a Sun pointing attitude to guarantee the necessary power generation from the solar array. Once acquired, the Sun pointing attitude is maintained for the whole mission using two different control algorithms: (1) a "coarse" Sun pointing attitude that makes use of a reduced set of sensors and actuators to maintain the orthogonality of solar incidence on the solar panel for a free-wobbling of the satellite pointing; and (2) a "fine" Sun pointing attitude, using the whole on-board capability to ensure an overall satellite pointing, accuracy better than 1 degree and the required attitude stability better than 0.1 degrees/s. The ACS ensures the pointing of the payload instrument towards a given direction always maintaining the Sun-satellite direction orthogonal to the solar panel surface. For AGILE, the near-orthogonality of the solar panel surface and the Sun-satellite direction is enforced within a few degrees of tolerance.

The AGILE satellite meets the 1-2 arcmin attitude reconstruction requirement by using two back-to-back oriented Star Sensors (S/S's), which are then an essential part of the satellite ACS. The star sensors (manufactured by Galileo Avionica) are equipped with on-board sky charts and software able to determine the satellite attitude. This information is used to obtain on board the sky coordinates for the gamma-ray individual events and for the Super-AGILE imaging system. To minimize the grammage surrounding the gamma-ray instrument, the S/S's are positioned in a back-to-back configuration in the "PL Unit" behind the solar panel and relatively far from the payload.

\subsection{Satellite timing and orbital positioning}

The AGILE satellite is equipped with a navigation transceiver that ensures an on-board timing accuracy within 2 microseconds. The navigation system information is first used on-board by the instrument DH unit to time tag individual events of the various detectors. This telemetry is also used by the ground segment to improve the satellite orbital tracking.

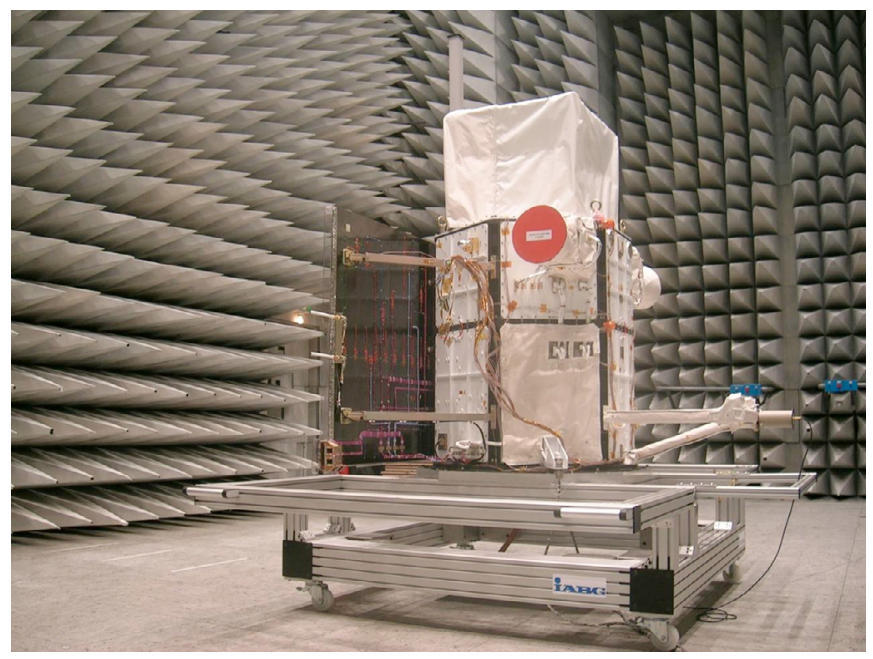

Fig. 12. The AGILE satellite during the qualification tests in IABG (Munich) (June 2006).

\subsection{Satellite communication and telemetry}

Communication with the ground station is ensured by an $S$-band transceiver that contacts the ASI ground station located in Malindi (Kenya) about 14 times a day, for a total visibility periods of about $160 \mathrm{mn}$ perday. During the visibility periods the $S$-band transmitter downloads the payload data, previously stored in the OBDH mass memory, with a net data rate of $500 \mathrm{kbps}$. The $S$-band transceiver is also used to upload the configuration telecommands necessary to the satellite operations.

The AGILE scientific instrument generates under normal conditions a telemetry rate of $\sim 50 \mathrm{kbit} \mathrm{s}^{-1}$. The satellite downlink rate for scientific telemetry is $512 \mathrm{kbit} \mathrm{s}^{-1}$. This rate is adequate for transmitting all the satellite and scientific data at every passage over the ground station.

The AGILE satellite also provides a special communication link dedicated to the fast transmission to the scientific community of basic information related to the on-board detection and processing of GRBs. This functionality is obtained using an ORBCOMM transceiver controlled by the spacecraft OBDH. The ORBCOMM transceiver is constantly connected to the ORBCOMM constellation (composed of 36 satellites in low Earth orbits) that is able to send a dedicated e-mail message to selected users as soon as a GRB is detected, independently from the baseline $S$-band ground station coverage. The AGILE ORBCOMM message to the ground provides the timing, coordinate reconstruction, and peak flux of the transient event.

\section{The Satellite qualification campaign}

The AGILE satellite carried out an extensive qualification campaign during the period 2006 June-July, with additional testing period during February-March, 2007. All AGILE satellite qualification tests were carried out at the IABG facility in Munich (Germany).

During the first campaign, an extended set of measurements and tests were carried out, including satellite mass and inertial measurements, acoustic tests, mechanical vibration tests, EMC tests, thermal vacuum, and thermal balance tests.

All the qualification tests were defined and performed according to an environmental test plan based on the launcher and orbit characteristics. In particular, thermal vacuum cycles were 
Table 2. Summary of instrument functional and scientific tests.

\begin{tabular}{ccc}
\hline \hline Activity & Facility & Date \\
\hline Payload AIV & Thales Alenia Space Italia, Milano (Italy) & Dec. 2004-Oct. 2005 \\
GRID Calibration & Laboratory Nazionali Frascati (Italy) & November 2005 \\
Satellite AIV & Carlo Gavazzi Space, Tortona (Italy) & Dec. 2005-May 2006 \\
Satellite Qualification & IABG, Munchen (Germany) & June-July 2006 \\
Scientific Configuration finalization & Carlo Gavazzi Space, Tortona (Italy) & Aug.-Sept. 2006 \\
Satellite refurbishment & Carlo Gavazzi Space, Tortona (Italy) & Oct.-Nov. 2006 \\
Satellite AIV post-refurbishment & Carlo Gavazzi Space, Tortona (Italy) & December 2006 \\
Super-A and MCAL-Burst calibrations & Carlo Gavazzi Space, Tortona (Italy) & January 2007 \\
Sat. Qualification post-refurbishment & IABG, Munich (Germany) & Feb.-March 2007 \\
Launch campaign & ISRO Space Center, Sriharikota (India) & April 2007 \\
\hline
\end{tabular}

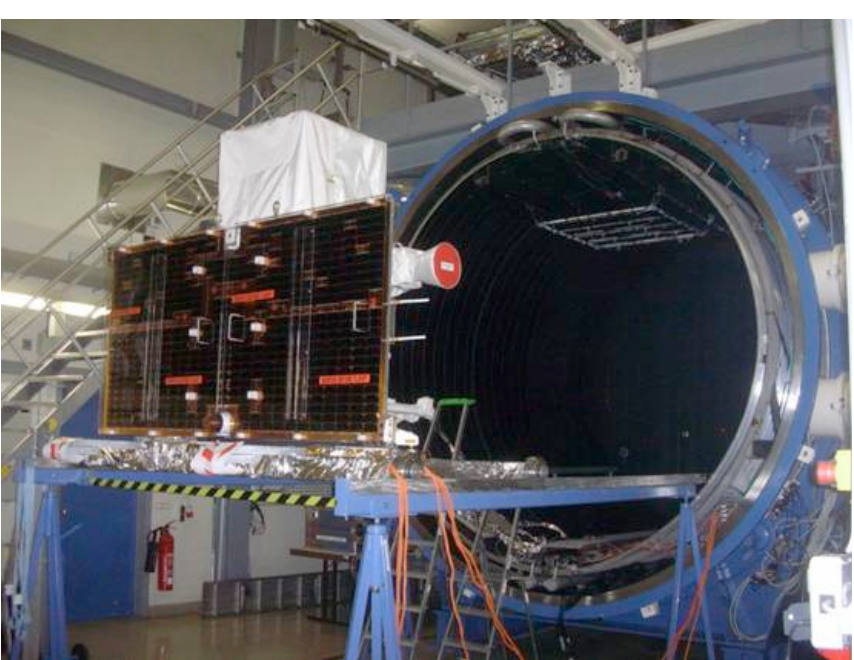

Fig. 13. The AGILE satellite being moved into the thermal-vacuum test chamber in IABG (Munich) (July 2006).

performed in the temperature ranges $(-20,+40)$ for the operative state, and $(-30,+50)$ for the non-operative state.

After all major individual tests, specific reduced functional tests were performed for both spacecraft and instrument. At the end of the qualification campaign, the test results were presented and discussed at the AGILE Test Review Board meeting.

Independently of the qualification outcome, for programmatic reasons the AGILE satellite was subject to an additional reworking during the period 2006 September-November. A significant reassembling of parts of the satellite was necessary to implement the substitution of a few components. An additional satellite qualification test phase was then carried out in February 2007 until the end of March. A complete satellite functional test was performed to verify the nominal operation of spacecraft and instrument subsystems including OBDH redundancy testing.

\section{The pre-launch AGILE scientific performance}

The scientific performance of the AGILE instrument has been checked during all the payload and satellite integration and testing phases. The tests carried out at the end of specific qualification sessions confirmed the nominal behavior of the instrument subsystems.

After having completed the satellite qualification tests, a series of additional final scientific measurements were performed, and their results were presented at the Mission Review Board. All detectors and subsystem functioning was judged to be nominal and within the scientific requirements and specifications.

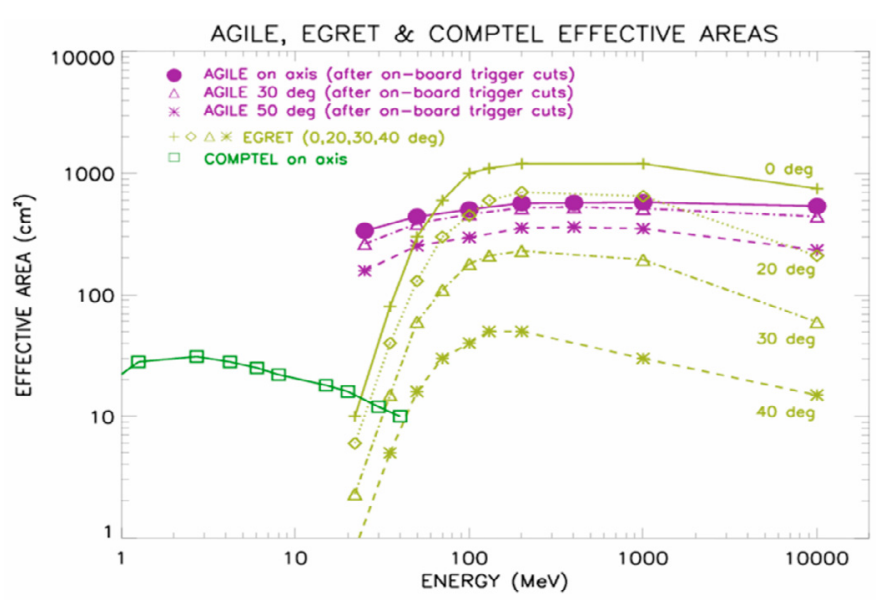

Fig. 14. The AGILE Gamma-Ray Imaging Detector (GRID) effective area as obtained by pre-launch simulations and instrument tests.

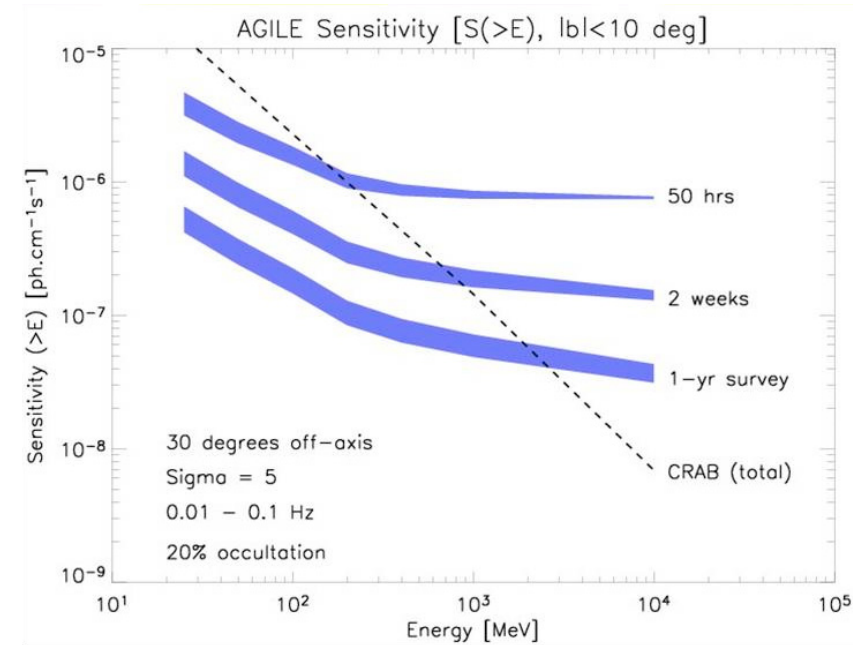

Fig. 15. Simulated integrated flux sensitivity of the AGILE-GRID as a function of energy for a 30-degree off-axis source in the Galactic plane. The Crab spectrum is shown by the dotted line.

The pre-launch overall scientific performance of the AGILE instrument detectors in terms of confirmed effective areas and sensitivities is summarized in Figs. 14-17 for the GRID, SuperAGILE, and MCAL detectors, respectively. 


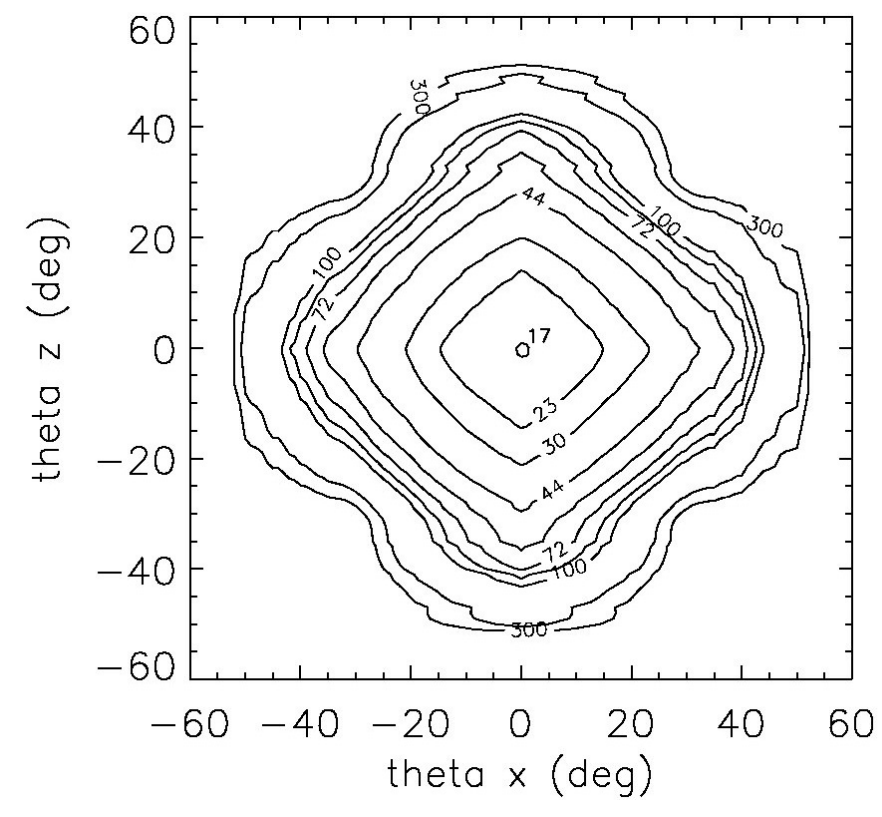

Fig. 16. Simulated sensitivity of Super-AGILE detector (all four units) expressed as (5 sigma) contour levels (in mCrab) over the field of view in the energy range $20-55 \mathrm{keV}$. Calculation carried out for a Crab-like spectrum, $50 \mathrm{ks}$ exposure.

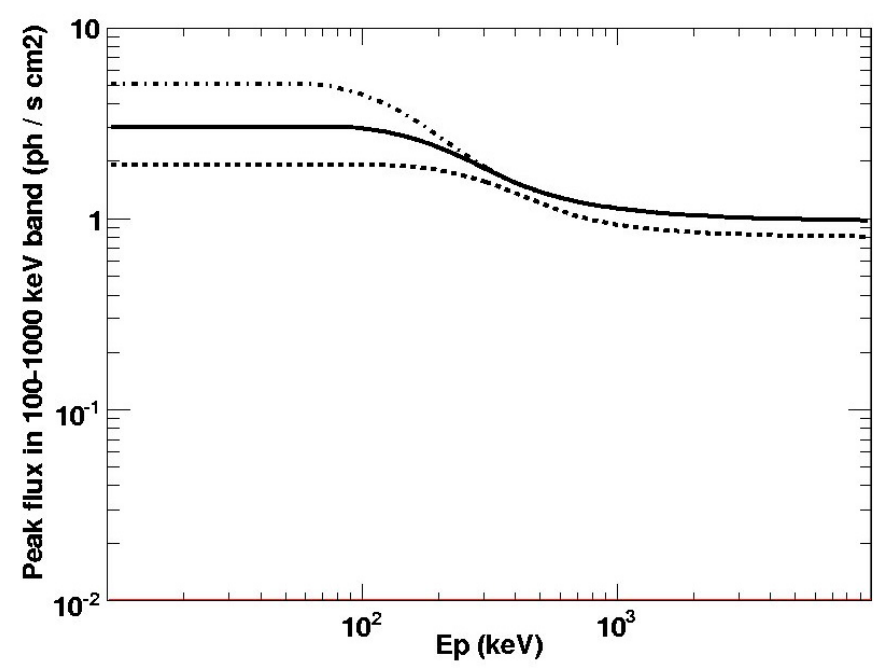

Fig. 17. MCAL sensitivity expressed as 3-sigma threshold peak flux of GRBs as a function of GRB peak energy at $30 \mathrm{deg}$ off-axis. Three different GRB spectra are modeled according to the Band model (Band et al. 1993). Continuous line: $\alpha=-1, \beta=-2.5$; dashed line $\alpha=-0.5$, $\beta=-2$; dot-dashed line $\alpha=-1, \beta=-3$.

\section{The AGILE ground segment: mission operation center}

The AGILE ground segment is an essential part of the Mission. It is divided into four components under ASI supervision and management:

- the ASI communication ground base in Malindi (Kenya);

- the Mission Operations Center (MOC), located at the Telespazio facility in Fucino (Italy);

- the AGILE Data Center (ADC), located at the ASI Science Data Center (ASDC) in Frascati (Italy).
In this section, we describe the AGILE Ground Segment (AGS) devoted to in-orbit operations. The AGILE Scientific Data Center is described in Sect. 12.

The satellite operations are carried out by the Ground Segment unit of Telespazio under the supervision of the ASI Mission Director. The AGILE Ground Segment is the main system in charge of satellite monitoring and control. The AGS is also in charge of the instrument observation planning and execution upon specific requests by the AGILE Team. The AGS main functions can be summarized as follows:

- satellite tracking and acquisition during the 14 passes per day over the TT\&C Malindi ground station;

- telecommand (TC) and telemetry (TM) management (TM acquisition, processing, display, archiving; TC generation, verification, and uplink);

- satellite orbit determination and propagation, satellite attitude determination, support to satellite ACS on-board operations;

- satellite sub-systems and payload monitoring and control, satellite modes of operations monitoring and control;

- mission planning generation and execution.

The instrument is continuously monitored and controlled with the bus satellite sub-systems. Scientific data are received by the Malindi ground station, transmitted to the Mission Operations Center (MOC) ground station in Fucino and locally archived there. Subsequently, typically within a few minutes, scientific data are transmitted to the ASI Scientific Data Center in Frascati. The AGILE Mission Operations Center is composed of the following main sub-systems: (1) the Satellite Control Center (SCC), (2) the Flight Dynamics Center (FDC), (3) the Mission Control Center (MCC), (4) the TT\&C ground station and communication network. Figure 18 shows a schematic summary of the AGILE GS.

The AGILE Satellite Control Center (SCC), located in Fucino, is in charge of all the satellite monitor and control functions, both in nominal and in contingency situations. The main SCC functions are the following: (1) satellite telemetry acquisition from TT\&C ground station, TM processing, display, and archiving; (2) handling of the satellite acquisition automatic procedures; (3) satellite database handling, and maintenance; (4) satellite sub-systems and payload health monitoring and control through housekeeping data presented on alpha-numeric and mimic displays; (5) real time or time-tagged telecommand preparation, TC to be uplinked to the satellite; (6) co-ordination with the TT\&C ground station for data and voice exchange and with reference to program track-mode antenna operations and to satellite tracking data reception to be processed by FDC; (7) communication network management.

The AGILE Flight Dynamics Center (FDC) carries out the satellite orbit determination and prediction, obtains the satellite attitude dynamics determination, and supports the satellite AGS on-board operations. The main FDC tasks are: (a) satellite performance monitoring; (b) attitude maneuver calculation for the pre-defined satellite pointing at scientific targets; (c) star tracker and navigation system receiver data handling. The AGILE FDC generates standard products including: (1) the Orbital Files, necessary for the satellite in-orbit operations and for the mission planning activity; (2) the Attitude Files, containing the reconstructed satellite attitude, necessary for the satellite in-orbit operations and for scientific activities support; (3) files containing the needed information to prepare the payload configuration tables to be uploaded (contact tables, SAA tables, occultation table, Earth vector table, star trackers table); (4) files containing 


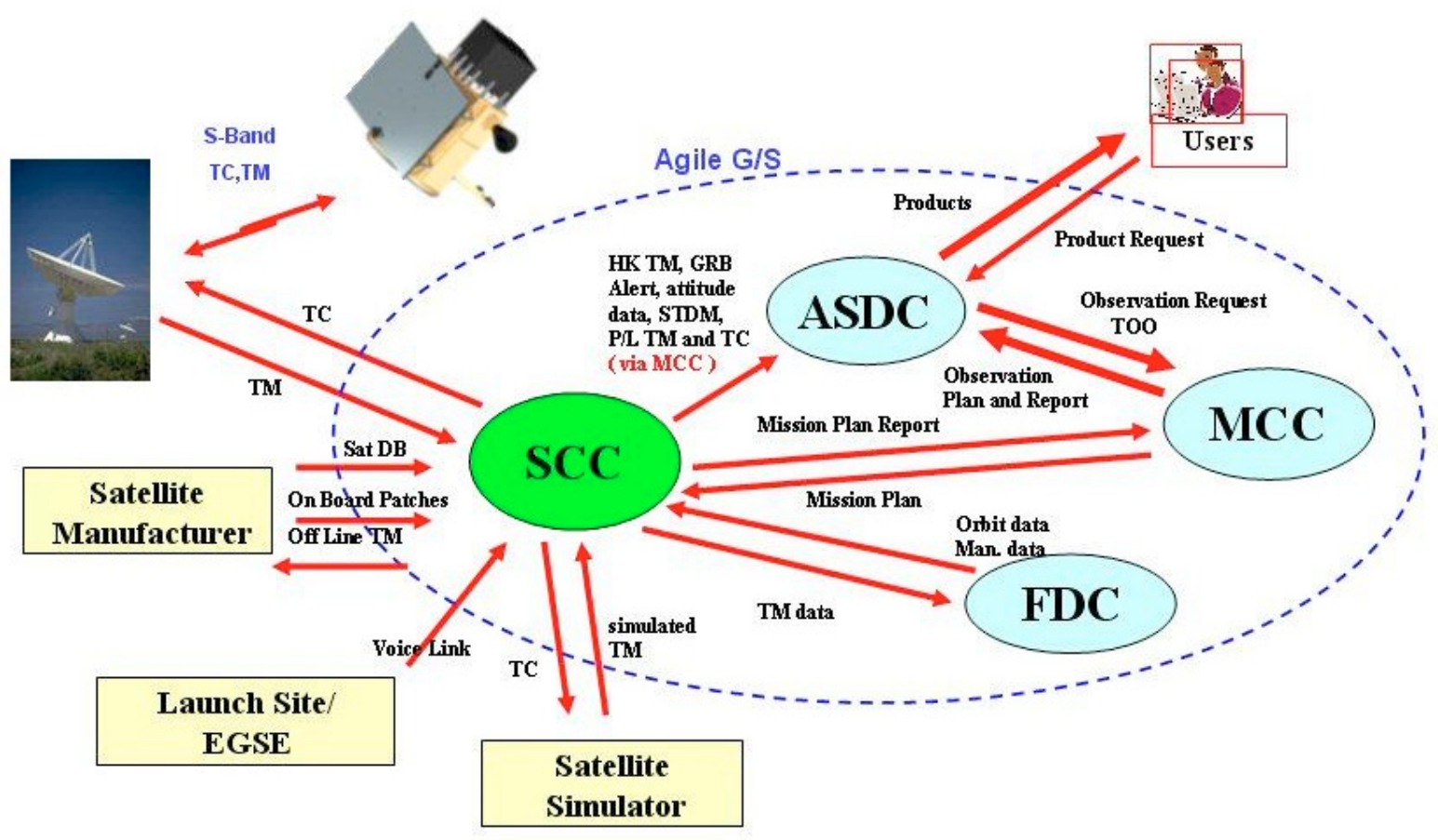

Fig. 18. A schematic view of the AGILE Ground Segment.

the information on parameters necessary to support the spacecraft activity; (5) the Orbital File for scientific purposes; (6) a Sequence of the Events file; (7) Two Line Elements (TLE) to be used for the TT\&C ground antenna Program Track mode.

The AGILE orbit determination is based on navigationsystem telemetry data processing and, as a backup, on AMD (Angular Measurement Data) measurements stored at TT\&C ground station with the antenna in auto-track mode.

The AGILE Mission Control Center (MCC), performs the mission planning and the instrument data handling management. In particular, the MCC is in charge of (1) the Payload scientific raw data archiving (level 0 archiving) and delivery to ASDC; (2) the Mission planning generation and preliminary check-out, according to the scientific observation requests; (3) full support for both the payload planning and the creation of command procedures to perform the AGILE flight operations.

The AGILE TT\&C ground station, based at the ASI Malindi (Kenia) Broglio Space Center, is in charge of $S$-band (S TX 2025 -2120 MHz e RX 2200-2300 MHz) RF ground-to-space satellite communication during all the AGILE mission phases. The main $\mathrm{TT} \& \mathrm{C}$ ground station tasks are the following:

- ground-to-space RF $S$-band interface with the satellite during all the mission phases;

- during satellite visibility over the station, telemetry is received from the satellite and telecommands (TCs) are uplinked to the satellite;

- real-time TM is extracted and sent to SCC, while off-line TM is locally stored to be sent to SCC at the end of the satellite pass;

- TCs received from SCC are uplinked to the satellite for immediate or time-tagged on-board execution;

- during satellite visibility over the station, the satellite tracking is performed, in "program track" or "auto-track" modes of operations;
- ground station equipment and configuration monitor and control.

The AGILE Communication Network, mainly based on the ASINET network provided by ASI, carries out the data and voice communication between the AGILE GS subsystems during all the mission phases. The AGILE Data Center located at ASDC is the ASI official interface with the AGILE GS during the satellite operations. It interacts with the MOC in submitting scientific requests of observations and receives the AGILE payload raw data from the AGS. In case of a GRB event it also receives notification.

The equatorial orbit of the AGILE satellite leads to satellite visibility every 90 minutes for a period of about 10 minutes. During each pass, the satellite receives telecommands from the Ground Station and downloads housekeeping and scientific telemetry. The MOC carries out the operational management of the satellite and the ground segment, guaranteeing the predefined observation plan provided by the AMB, and also guaranteeing the delivery of the scientific data to ASDC.

To guarantee the correct management of the in-orbit operations with respect to the mission safety requirements, the MOC defines operational procedures and operational strategies allowing command of the satellite operations for nominal and contingency phases. The sequence of commands, prepared by the Mission Control Center (MCC), are to be sent in a "time-tagged" format, using the satellite on-board storage ability. In case of onboard anomalies, the satellite is able to self-configure in a "safe" state that guarantees its surviving for at least $72 \mathrm{~h}$.

\section{The launch campaign and orbital parameters}

After completion of all Flight and Ground Segment qualification tests, the AGILE satellite was transferred from Munich (Germany) to the ISRO Sriharikota base near Chennai (India) 


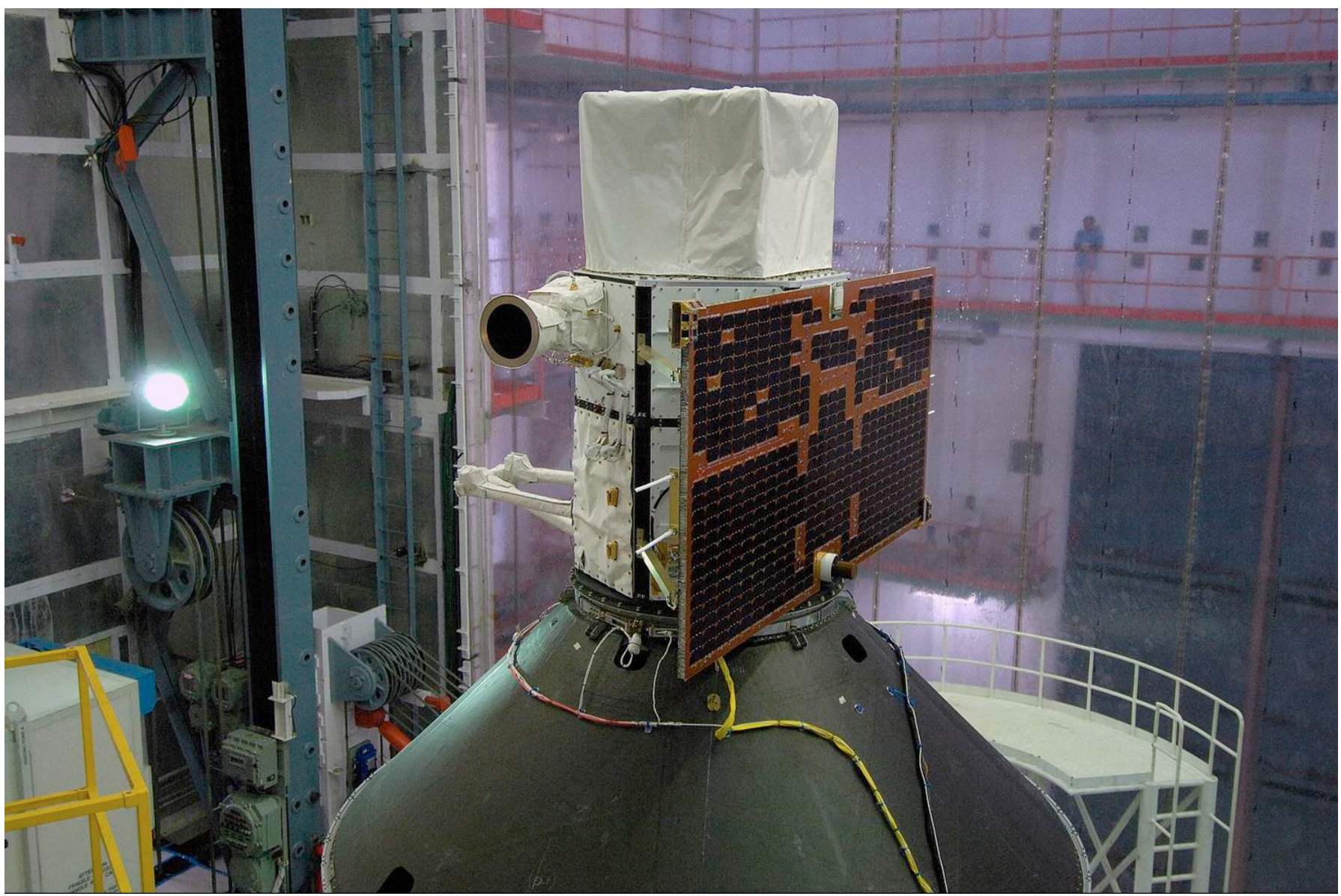

Fig. 19. The AGILE satellite integrated with the fourth stage of the PSLV C-8 rocket in the ISRO Sriharikota launch base (April 15, 2007).

at the end of March 2007. The AGILE launch campaign in Sriharikota started on April 1 and ended with a very successful launch on 2007 April 23.

\subsection{The launch campaign}

A series of functional and reduced scientific tests were carried out in the Sriharikota satellite integration facility during the period 2007 April 2-9. A rehearsal of the complete test procedure of the in-orbit commissioning of the satellite was performed. These tests included the execution of all baseline satellite in-orbit procedures. Spacecraft and instrument commandability were extensively checked.

An example of the AGILE pre-launch measurements carried out during the AGILE launch campaign is shown in Fig. 20. The plot shows a sequence of spectral measurements obtained with the AGILE MCAL for different environmental and testing conditions. The data points marked in green were obtained during the satellite-launcher pre-integration tests at the Sriharikota facility. The data marked in blue were obtained during the final pre-launch tests with the satellite fully integrated with the PSLV$\mathrm{C} 8$ at a height of about $50 \mathrm{~m}$ above the ground. In this case, the MCAL threshold was set near $600 \mathrm{keV}$. Note the difference of the pre- and post-integration spectra that we interpret as caused by different natural radioactive background conditions.

The AGILE satellite integration with the PSLV-C8 launcher started on April 12 and was successfully completed on 2007 April 17. At the end of this integration phase and before the final fairing assembly completion, a reduced set of functional and

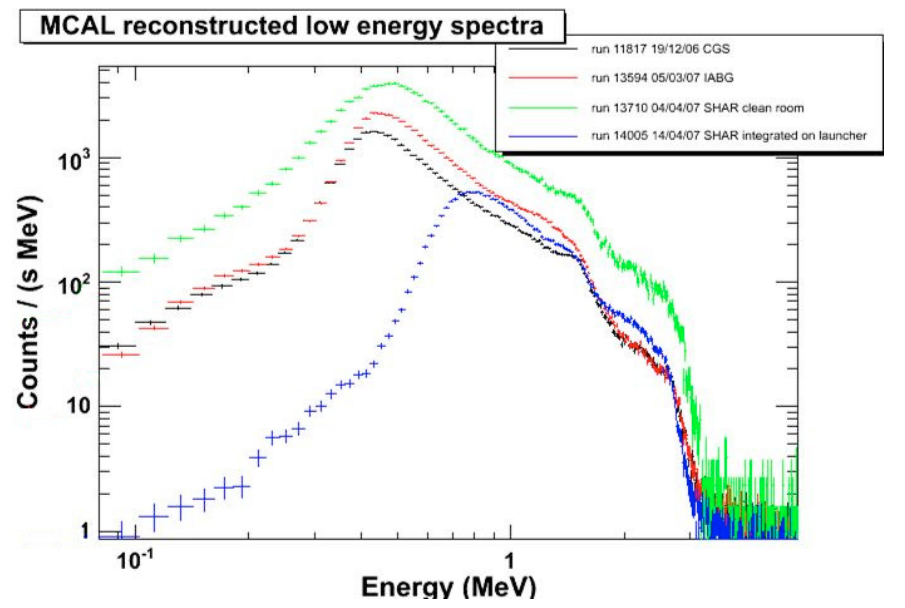

Fig. 20. Sequence of spectral measurements obtained with the AGILE MCAL for different environmental and testing conditions. Black data points: data obtained during the satellite integration at the CGS facility in Tortona (Italy). Red data points: data obtained during the satellite qualification at the IABG facility in Munich (Germany). Green data points: data obtained during the satellite launcher pre-integration tests at the Sriharikota facility. Blue data points: data obtained during the final pre-launch tests with the satellite fully integrated with the PSLV$\mathrm{C} 8$ at a height of about $50 \mathrm{~m}$ above the ground.

scientific tests were performed. The integrated PSLV-C8 rocket was transported to the launch pad on 2007 April 18: the launch countdown started on 2007 April 20. 
Table 3. AGILE orbital parameters.

\begin{tabular}{ll}
\hline \hline Orbital parameter & \\
\hline Semi-major axis & $6,922.5 \mathrm{~km}$ \\
Average altitude & $535 \mathrm{~km}$ \\
Eccentricity & 0.002 \\
Inclination angle & 2.47 degrees \\
Apogee altitude & $553 \mathrm{~km}$ \\
Perigee altitude & $524 \mathrm{~km}$ \\
\hline
\end{tabular}

On 2007 April 23 at 12:00 noon local time, the PSLV-C8 rocket was successfully launched with a nominal performance. After about $20 \mathrm{mn}$, the fourth stage (with the AGILE satellite still integrated) performed the inclination correction maneuver and achieved the nominal orbit for the mission with the required height and inclination. Satellite and fourth-stage separation occurred immediately afterwards.

The first contact with the Malindi ground station occurred nominally at the first satellite's first pass over Kenya, about $75 \mathrm{mn}$ after launch.

\subsection{Orbital parameters}

The AGILE orbit is quasi-equatorial, with an inclination of 2.5 degrees and average altitude of $535 \mathrm{~km}$. A small inclination low-earth orbit (LEO) is a clear plus for our mission because of the reduced particle background (as immediately verified in orbit by all instrument detectors). Furthermore, a low-inclination orbit optmizes the use of the ASI communication ground base at Malindi (Kenya). Table 3 provides the final AGILE orbital parameters. Depending on solar activity, the AGILE satellite re-entry in the atmosphere is predicted to happen not earlier than 2012.

\section{Early operations in orbit}

After the nominal launch and the correct satellite attitude stabilization within approximately 2 days, the operations focused on two different tasks: (1) the commissioning of both the satellite platform and instrument; (2) in-orbit scientific calibration of the instrument. We briefly describe here these two main activities, postponing a detailed description to forthcoming publications.

\subsection{The satellite in-orbit commissioning and instrument checkout phase}

The satellite platform was tested and functionally verified in all its main capabilities during the last days of April. The checkout sequence of tests ended with the satellite fine-pointing attitude finalization that implies the nominal $\sim 1$ degree pointing accuracy and the 0.1 degree/sec stabilization. Attitude reconstruction, both on-board and on the ground, was tested to be initially within a few arcminutes accuracy. A sequence of early pointings was carried out, typically lasting for a few days.

The instrument subsytem switch-on started in early May and proceeded with nominal behavior of all detectors. A first check of the instrument housekeeping telemetry indicated a nominal particle background rate as predicted by extensive simulations (Cocco et al. 2002; Longo et al. 2002). Figure 21 shows a typical background count rate on a lateral AC panel throughout the equatorial orbit.

The GRID acquisition rate after a complete on-board processing and Earth gamma-ray albedo rejection turned out to be

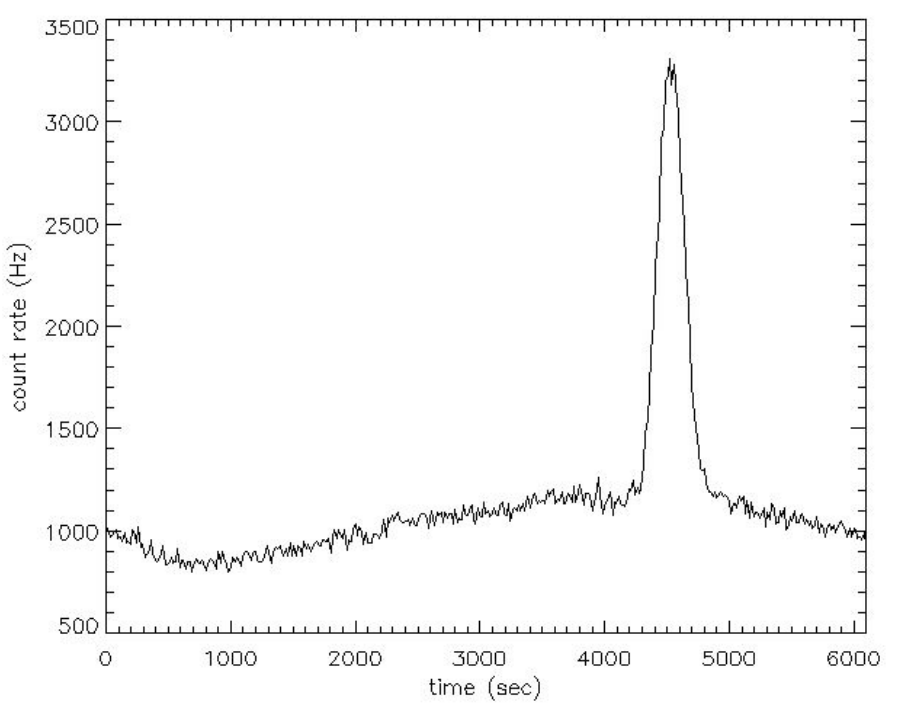

Fig. 21. AGILE AC count rate throughout the whole orbit for one of the 12 lateral anticoincidence panels. The count rate peak occurs in coincidence with the passage over the South Atlantic Anomaly.

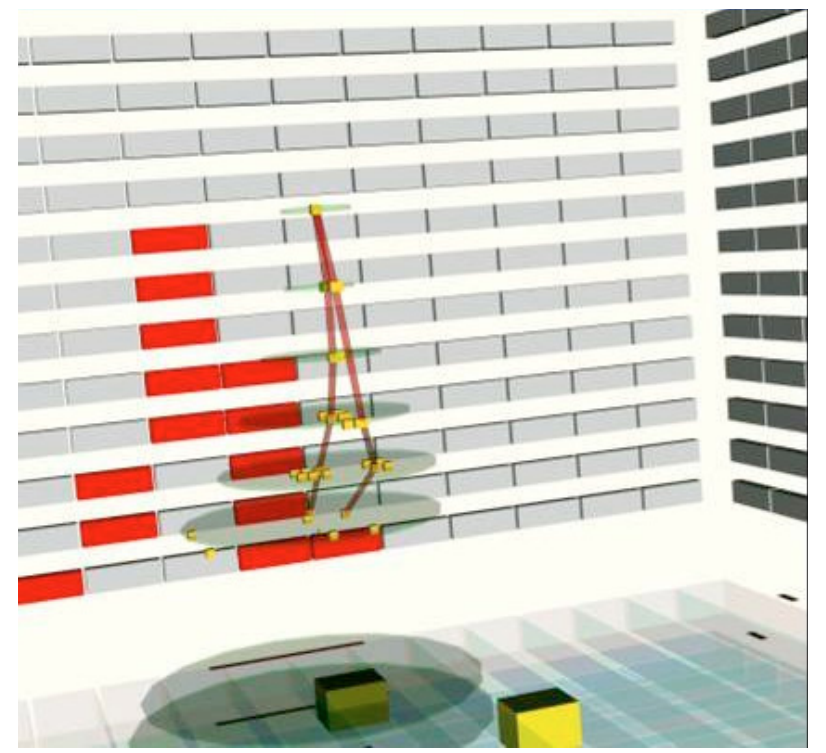

Fig. 22. The first gamma-ray event detected by AGILE in space (2007 May 10).

stable throughout the orbit with a modulation induced by the Earth sweeping the GRID FOV (within 1-7 Hz). A detailed account of the GRID and overall on-board scientific telemetry will be reported elsewhere (Argan et al. 2008a). The GRID subsystem was extensively tested first, and the baseline trigger logic photon acquisition started on May 10. Figure 22 shows the first gamma-ray photon detected by the GRID and transmitted to the ground as the first event of the first telemetry packet. The overall particle background turned out to be within the expected rate, both at the level of the anticoincidence rate, and especially at the so called Level-1 and Level-2 event processing (Argan et al. 2008a).

The Super-AGILE detector was tested immediately afterwards with a dedicated pointing of an extragalactic field. A detailed scan and test of the individual SA strip thresholds was carried out with an optimized parameter stabilization procedure (Pacciani et al. 2008). Hard X-ray data were obtained with a nominal performance and very low background. These 


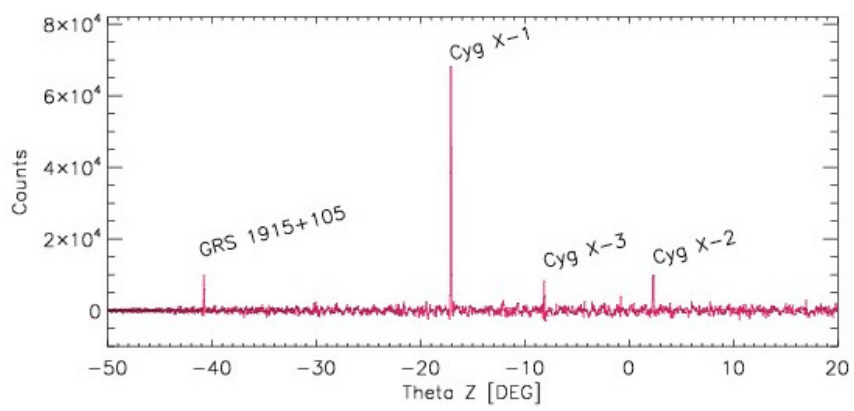

Fig. 23. Super-AGILE deconvolved sky image (one detector unit) of the Galactic plane obtained on 2008 June 2 (total effective exposure of $45 \mathrm{ks}$ ). Several hard X-ray sources are detected as marked in the figure (positions given in detector coordinates).

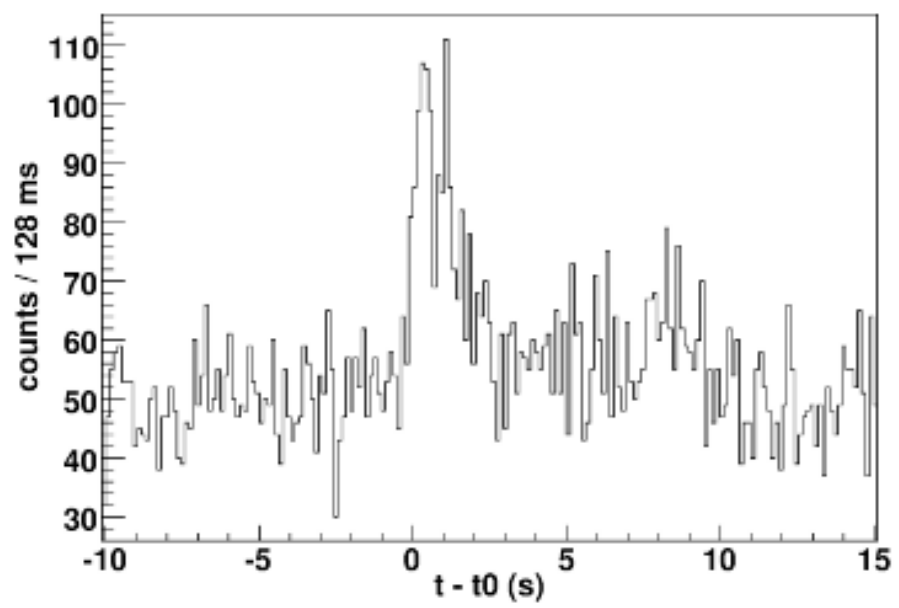

Fig. 24. The lightcurve (128 msec time bin) of GRB 080319C (Pagani et al. 2008; Marisaldi et al. 2008) detected by the AGILE minicalorimeter in the energy band $0.3-5 \mathrm{MeV}$.

operations ended successfully in mid-July 2007 (Feroci et al. 2008). Figure 23 shows an example of a typical 1-day pointing in the Galactic plane with the detection of Cyg X-1, Cyg X-2, Cyg X-3, and GRS 1915+105.

The MCAL detector optimization and configuration checkout was performed in parallel with other instrument testing. A satisfactory detector configuration was obtained at the end of June 2007 (Marisaldi et al. 2008b). Figure 24 shows an example of a typical GRB detected by the MCAL in the energy range $0.3-5 \mathrm{MeV}$.

\subsection{In-orbit instrument calibration}

An account of the AGILE pointings and in-orbit calibration details will be presented elsewhere. We briefly summarize here the main outcomes of the early in-orbit calibration phase.

Preliminary gamma-ray data obtained for the Vela pulsar in early June 2007 confirmed immediately the good quality of the GRID background rejection and its imaging capability. The scientific operations started in early July 2007 with a 2month observation of the Vela pulsar region. At the end of August 2007 AGILE devoted about 1 week to a pointing of the Galactic center region. Finally, AGILE carried out the gammaray and hard X-ray calibration with the Crab pulsar during the months of September and October 2007. Figure 25 shows a 1-day integration of the gamma-ray sky of the Galactic anticenter region(containing the Crab, Geminga, as well as the Vela

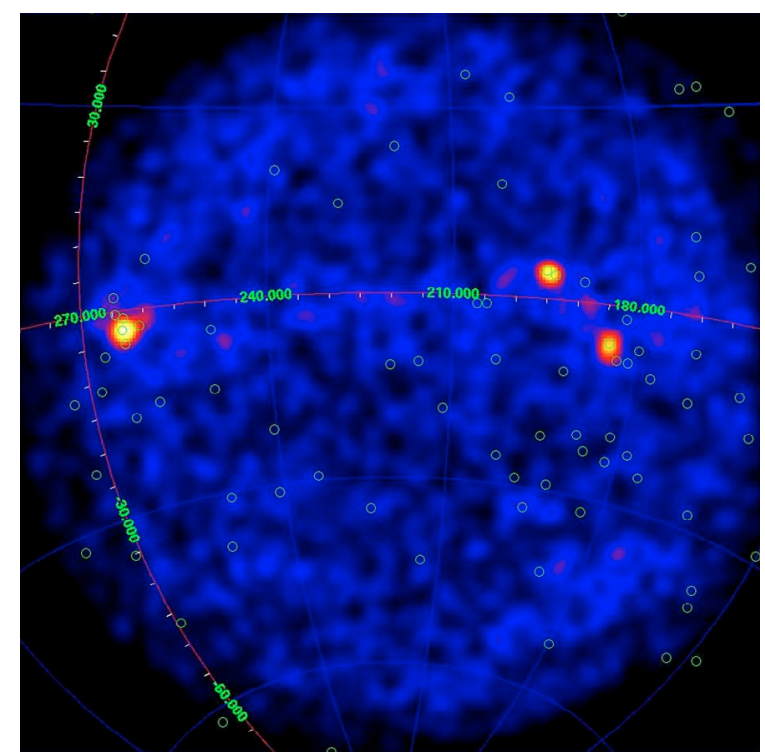

Fig. 25. The AGILE-GRID 1-day gamma-ray counts map for photons above $100 \mathrm{MeV}$ obtained on 2007 September 28. The unprecedently large FOV includes for the first time in a single map all three of the most important gamma-ray pulsars: Vela, Crab, and Geminga.

gamma-ray pulsars). This field was repeatedly observed with 1-day pointings with the Crab pulsar position at different angles with respect to the instrument axis. The in-orbit calibration phase was successfully completed at the end of October 2007 achieving a good performance for both the gamma-ray and hard $\mathrm{X}$-ray imagers.

We consider here the GRID effective area as obtained from our calibration measurements of the Vela PSR. This pulsar was repeatedly observed by AGILE at different off-axis angles during the first year of operations. As an example of the GRID typical values of the gamma-ray effective area vs. photon energy obtained within the field of view, we show in Fig. 26 the results between $70 \mathrm{MeV}$ and $\sim 1 \mathrm{GeV}$ as checked with real data and simulations obtained for the Vela PSR at an off-axis angle of 30 degrees. For the Vela PSR gamma-ray spectrum, we used the EGRET measurements as reported in Thompson et al. (1998). We notice good agreement between expectations and real inorbit performance of the GRID.

Super-AGILE calibration data in orbit were obtained pointing at the Crab PSR for a variety of zenithal and roll angles. Postponing a detailed description of the calibration campaign to a dedicated paper, we can confirm the good agreement between the achieved real sensitivity and the simulations shown in Fig. 16. MCAL typically detects one GRB per week Marisaldi et al. (2008c). Figure 27 shows the in-orbit performance of MCAL as tested from the detection of selected GRBs. The scientific performance of the AGILE instrument as tested in orbit is therefore nominal for all subsystems and detectors.

The nominal science verification phase (SVP) observations were interrupted for specific pointings that were carried out either for planned multifrequency campaigns (for 3C 279 and 3C 273 in early July, 2007) or reacting to external alerts due to relevant optical activity of blazars (3C 454.3 in mid-July 2007; TXS $0716+714$ at the end of October 2007, W Comae in June 2008). The AGILE Cycle-1 program of nominal scientific observations started on 2007 December 1. 


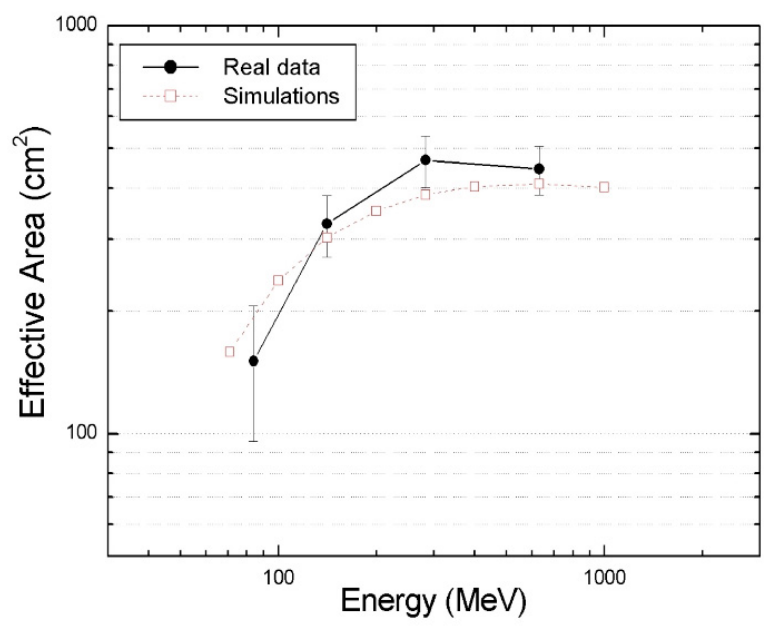

Fig. 26. AGILE-GRID effective area at 30 degrees off-axis as a function of photon energy. Open squares: results of numerical simulations; filled circles: values obtained by comparing AGILE data with EGRET measurements of the Vela PSR.

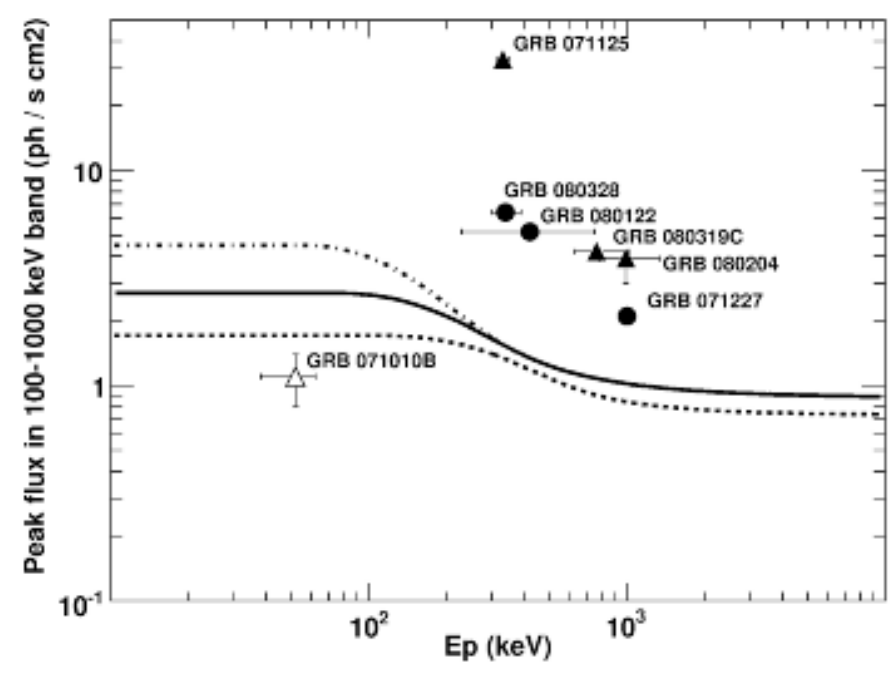

Fig. 27. MCAL in-orbit detection of selected GRBs represented in a diagram showing peak flux vs. GRB peak energy. 3-sigma thresholds are shown for three different GRB spectra models (using symbols as in Fig. 17). Solid triangles: GRBs detected by MCAL at incident angle $<90^{\circ}$. Solid circles: GRBs detected by MCAL at incident angle $>90^{\circ}$. Hollow triangle: GRB at incident angle $<90^{\circ}$ but not detected by MCAL (GRBs spectral parameters are derived from KonusWind and Suzaku-WAM GCNs).

\section{The AGILE data center}

AGILE scientific data (about 300 Mbit/orbit) are telemetered from the satellite to the ASI ground station in Malindi (Kenya) at each satellite passage (approximately every $95 \mathrm{mn}$ ). A fast ASINET connection between Malindi and the Satellite Control Center at Fucino and then between Fucino and the ASI Science Data Center (ASDC) in Frascati ensures the data transmission every orbit.

Scientific data storage, quicklook analysis, and management of the AGILE Guest Observer Program are carried out at the
ASDC. After pre-processing, scientific data (level-1) are corrected for satellite attitude data and processed by a dedicated software. GRID background rejection and photon-list determination are the main outputs of this first stage of processing. Level-2 data are produced for a full scientific analysis.

Gamma-ray data generated by the GRID are analyzed by a dedicated special software producing: (1) sky-maps, (2) energy spectra, (3) exposure, (4) point-source analysis products, and (5) diffuse gamma-ray emission. This software is aimed to allow the user to perform a complete science analysis of specific pointlike gamma-ray sources or candidates, as well as a timing analysis. This software is also available for the guest observers.

Super-AGILE data are deconvolved and processed to produce 2-D sky images through a correlation of current and archival data of hard X-ray sources. Super-AGILE dedicated software produce lightcurves, spectra, and positioning of sources detected in the hard X-ray (18-60 keV).

The AGILE data processing main tasks carried out at the ADC can be summarized as follows:

- Quicklook Analysis (QLA) of all gamma-ray and hard $\mathrm{X}$-ray data, aimed at a fast scientific processing (within a few hours/1 day depending on source intensity) of all AGILE scientific data.

- web availability of QLA results to the international community for alerts and rapid follow-up observations (http://agile.asdc.asi.it,http://agile. iasf-roma.inaf.it);

- GRB positioning and alerts through the AGILE Fast Link, capable of producing alerts within 10-30 mn after trigger;

- standard science analysis of specific gamma-ray sources open to the AGILE Guest Observer Program;

- web availability of the standard analysis results of the hard X-ray monitoring program by Super-AGILE.

\section{The AGILE scientific management}

The Mission management is based on:

- the AGILE Mission Board (AMB);

- the AGILE Mission Directorate;

- the AGILE Users Committee;

\subsection{The AGILE mission board}

The AGILE Mission Board is the executive Board overseeing all Mission operations and is entitled to make final decisions regarding all scientific and technical issues.

The AMB is composed by the Principal Investigator (Tavani), the co-Principal-Investigator (Barbiellini), two ASIappointed scientists (Giommi \& Colafrancesco), and by the AGILE Mission Director (Salotti). The AMB approves the satellite scheduling, decides on repointings and Mission-ofOpportunity observations, and decides on issues regarding the AGILE Guest Observer Program.

\subsection{The AGILE mission directorate}

The ASI appointed AGILE Mission Director (MD) oversees all satellite operations and decides about all technnical issues regarding the optimal satellite operations, and pointings. 


\subsection{The AGILE users committee}

The AGILE Users Committee (AUC) is a 5-member group of scientists not associated with the AGILE Team. The AUC advices the AGILE Mission Board on issues regarding the satellite pointing strategy and data access to the astrophysical community.

\subsection{The AGILE pointing program}

The AGILE Pointing Plan is determined according to the Mission scientific program as proposed by the AGILE Team to the AMB after the recommendations of the AUC. Solar panel constraints determine the observability of a given sky region depending on the season.

\subsubsection{Baseline pointings}

The Cycle-1 of AGILE scientific observations started on December 1, 2007 and will last one year. The Cycle-1 is based on a set of pre-established baseline pointings approved by the AMB. A detailed pointing schedule of the AGILE Cycle-1 phase can be found in http://asdc.asi.it.

\subsubsection{Satellite repointings}

AGILE can react to transient events of special relevance occurring outside the current pointing FOV by properly re-pointing the satellite (always satisfying the solar panel constraints). The re-pointing has to be approved by the AGILE Mission Board and $\mathrm{MD}$, and a special procedure is implemented by the MOC to re-orient the satellite within the shortest possible time. Typical satellite maneuver timescales are approximately 1-2 orbits.

For important transients detected within the AGILE-GRID and not in the Super-AGILE FOV, minor re-pointings (20-30 degrees) are envisioned to allow the coverage of the gamma-ray transient also by the X-ray imager.

Drastic re-pointings requiring a major re-orientation of the AGILE satellite are treated as target-of-opportunity (ToO) observations and are foreseen for events of major scientific relevance detected by other observatories.

\section{The AGILE guest observer program}

AGILE is a Small Scientific Mission with a science program open to the international scientific community. A fraction of the AGILE gamma-ray data are available to the AGILE guest observer program (GOP) that is open to the international community on a competitive basis. The AGILE Cycle-1 GOP started in 2007 December and ended on 2008 November 30. The 1-year Cycle-2 started on 2008 December 1. Details about the AGILE scientific programme can be found in http://wWw . asdc. asi. it.

\section{The AGILE multiwavelength program}

The scientific impact of a high-energy mission such as AGILE (broad-band energy coverage, very large fields of view) is greatly increased if an efficient program is carried out for fast followup and/or monitoring observations by ground-based and space instruments. The AGILE Science Program overlaps with and is complementary to those of many other high-energy space Missions (INTEGRAL, RXTE, XMM-NEWTON, CHANDRA,
SWIFT, SUZAKU, RHESSI, FERMI) and ground-based instrumentation (radio telescopes, optical observatories, TeV observatories). The AGILE Science Program potentially involves a large astronomy and astrophysics community and emphasizes quick reaction to transients and a rapid communication of crucial data. Past experience has shown that, because of a lack of fast reaction to gamma-ray transients (within a few hours/days for unidentified sources,)many gamma-ray sources could not be identified. AGILE takes advantage of the combination of its gamma-ray and hard X-ray imagers. Several working groups are operational on a variety of scientific topics including blazars, GRBs, pulsars, and Galactic compact objects. The AGILE Team, together with the ASDC, is open to collaborations with multifrequency observing groups.

Acknowledgements. The AGILE program has been developed under the auspices of the Italian Space Agency with co-participation of the Italian Institute of Astrophysics (INAF) and of the Italian Institute of Nuclear Physics (INFN). A very important support to the project has been provided by CNR and ENEA. The scientific research carried out for the project has been partially supported under the grants ASI-I/R/045/04 and ASI-I/089/06/0,1,2. We acknowledge the crucial programmatic support of the former ASI Scientific Director and ASI President G.F. Bignami, of the ASI Presidents S. De Julio and S. Vetrella, as well as of the former CNR President (L. Bianco), the former INAF President (P. Benvenuti), the former INAF Special Commissioner (S. De Julio), and the current INAF (T. Maccacaro) and INFN (R. Petronzio) Presidents.

The ASI Director General (L. De Magistris) provided crucial support for the Mission. Several ASI personnel supported the Mission in different phases and conditions. We mention here P. Cecchini, S. Di Pippo, D. Frangipane, P. Caporossi, M. Cosmo, Q. Rioli, C. Musso.

INAF and INFN directorates and President offices offered a very important support for the Mission. We are particularly grateful to the Director of the INAF Projects Office (P. Vettolani), the INFN Directorate member (B. D'Ettorre Piazzoli), and INFN Commission-2 Director (F. Ronga). We recognize the critical help and unfailing support offered by the Directors G. Villa, D. Maccagni, R. Mandolesi, P. Ubertini, A. Vacchi.

A large number of scientists and engineers contributed to the success of the Mission in a substantial way at different stages of the project. We mention here the executive Directors L. Zucconi, M. Muscinelli, A. Beretta, R. Aceti, F. Longoni, R. Cordoni, R. Starec, and the managers G. Cafagna and R. Terpin. We also thank N. Kociancic, P. Bresciani. A. Ercoli-Finzi supported and offered advice at critical phases of the project. A particular recognition is given to the very delicate task carried out by G. Grossi. We also thank the IABG management (Munich) and in particular A. Grillenbeck.

We warmly thank the INFN-LNF staff for invaluable support and help during the 2005 November AGILE calibration campaign. In particular we thank the LNF Director M. Calvetti, and G. Mazzitelli, P. Valente, M. Preger and P. Raimondi. Special recognition is to be given to the outstanding technical and management performance of the ISRO personnel during the AGILE launch campaign in India. Special thanks go to the ISRO Sriharikota base Director A. Nair and to the PSLV C-8 launch Director N. Narayanamoorthy and his very skilled team. We acknowledge the collaborative support of the ANTRIX Director K.R. Sridhara Murthi, and of D. Radhakrishnan.

Strong support to the Mission has been provided by the CIFS Scientific Secretariat (G. Ardizzoia), and by the AGILE Team Scientific Secretariat members, C. Mangili, B. Schena. We acknowledge the skills and the effective collaboration of the current AGILE Team Secretariat, E. Scalise, and L. Siciliano. Personnel of the CNR, INAF and INFN administrations were (and are) very important for the success of the mission. We thank in particular the administrations of INAF-IASF Rome, Milan, and Bologna for their support. Updated documentation on the AGILE Mission can be found at the web sites http://www.asdc.asi.it, and http://agile.iasf-roma.inaf.it.

\section{References}

Aharonian, F., Akhperjanian, A. G., Bazer-Bachi, A. R., et al. 2006, ApJ, 636, 777

Argan, A., et al. 2004, Proc. IEEE-NSS, 1, 371

Argan, A., et al. 2008a, A\&A, submitted

Argan, A., et al. 2008b, NIM, submitted

Band, D., Matteson, J., Ford, L., et al. 1993, ApJ, 413, 281

Bakaldin, A., Morselli, A., \& Picozza, P., et al. 1997, Astrop. Physics, 8, 109 
Barbiellini, G., Boezio, M., Candusso, M., et al. 1995a, Nuclear Physics B, 43, 253

Barbiellini, G., Boezio, M., Casolino, M., et al. 1995b, Nucl. Instrum. \& Methods, 354, 547

Barbiellini, G., Tavani, M., Budini, G., et al. 2000, Proceedings of the 5th Compton Symposium, AIP Conf. Proceedings, ed. M. McConnell, 510, 750

Barbiellini, G., Fedel, G., Liello, F., et al. 2002, NIM A, 490, 146

Bhat, C. L., Fishman, G. J., Meegan, C. A., et al. 1992, Nature, 359, 217

Bignami, G. F., Fichtel, C. E., Hartman, R. C., \& Thompson, D. J. 1979, ApJ, 232, 649

Casandjian, J.-M., \& Grenier, I. A. 2008, A\&A, submitted [arXiv:0806.0113]

Cocco, V., Longo, F., \& Tavani, M. 2002, NIM A, 486, 623

Cousins, D. J., Guénault, A. M., Pickett, G. R., et al. 1995, ApJS, 101, 259

Del Monte, E., Soffitta, P., Morelli, E., et al. 2007, NIM A, 572, 708

Donnarumma, I., Costa, E., Del Monte, E., et al. 2006, SPIE, 6266, 626636

Donnarumma, I., Pacciani, L., Lapshov, I., et al. 2008, Appl. Opt. (in press)

Esposito, J. A., Hunter, S. D., Kanbach, G., \& Sreekumar, P. 1996, ApJ, 461, 820.

Evangelista, Y., Costa, E., Del Monte, E., et al. 2006, SPIE, 6266, 626635

Evangelista, Y., Costa, E., Del Monte, E., et al. 2008, SPIE (in press)

Feroci, M., Costa, E., Soffitta, P., et al. 2007, NIM. A, 581, 728

Feroci, M., Costa, E., Soffitta, P., et al. 2008, SPIE, in press

Fichtel, C. E., \& Trombka, J. I. 1997, Gamma-Ray Astrophysics, NASA Reference Publication no. 1386, September 1997

Fichtel, C. E., Hartman, R. C., Kniffen, D. A., et al. 1975, ApJ, 198, 163

Fuschino, F., Labanti, C., Galli, M., et al. 2008, NIM A, 588, 17

http://glast.gsfc.nasa.gov, htpp://glast. stanford.edu

Hartman, R. C., Bertsch, D. L., Bloom, S. D., et al. 1999, ApJS, 123, 279

Kramer, M., Bell, J. F., Manchester, R. N., et al. 2003, MNRAS, 342, 1299

Kraushaar, W. L., Bell, J. F., Manchester, R. N., et al. 1972, ApJ, 177, 341

Labanti, C., Marisaldi, M., Fuschino, F., et al. 2006, Proc. SPIE, 6266, 62663

Labanti, C., et al. 2008, Nucl. Inst. Meth. A., in press

Longo, F., Cocco, V., \& Tavani, M. 2002, NIM A, 486, 610

Longo, F., et al. 2008, in preparation

Marisaldi, M., Fornari, F., Labanti, C., et al. 2008a, GRB Coordinates Network 7457

Marisaldi, M., et al. 2008b, SPIE, in press

Marisaldi, et al. 2008c, A\&A, accepted [arXiv:0809.3917]

Mayer-Hasselwander, et al. 1979, Annals of the New York Academy of Sciences, Proc. of the Ninth Texas Symp., 226, 211

Pacciani, L., et al. 2008, NIM A, in press

Pagani, C., Barthelmy, S. D., Baumgartner, W. H., et al. 2008, GRB Coordinates Network 7442

Perotti, F., Fiorini, M., Incorvaia, S., et al. 2006, NIM A, 556, 228

Prest, M., Barbiellini, G., Bordignon, G., et al. 2003, NIM A, 501, 280

Pucella, F., et al. 2008, in preparation

Schnied, E. J., Bertsch, D. L., Catelli, J. R., et al. 1996, in AIP Conf. Proc., 384, 253

Sturner, S. J., \& Dermer, C. D. 1995, A\&A, 293, 17

Swanenburg, B. N., et al. 1981, ApJ, 242, L69

Tavani, M. 2003, Texas in Tuscany, XXI Symposium on Relativistic Astrophysics, Florence, Italy, 9-13 December 2002, 183
Tavani, M., Mukherjee, R., Mattox, J. R., et al. 1997, ApJ, 479, L109

Tavani, M., Barbiellini, G., Caraveo, P., et al. 1998, AGILE Phase A Report

Tavani, M., Barbiellini, G., Budini, G., et al. 2000, Proceedings of the 5th Compton Symposium, AIP Conf. Proc., ed. M. McConnell, 510, 746 Tavani, M., Barbiellini, G., Argan, A., et al. 2008a, NIM A, 588, 52

Tavani, M., et al. 2008b, NIM, submitted

Thompson, D. J., Bertsch, D. L., Fichtel, C. E., et al. 1993, ApJS, 86, 629

Thompson, D. J., Bertsch, D. L., Dingus, B. L., et al. 1996, ApJS, 107, 227

Thompson, D. J., et al. 1998, in Proc. 4th CGRO Symp., AIP Conf. Ser., 410, 39

Vercellone, S., Soldi, S., Chen, A. W., et al. 2004, MNRAS, 353, 890

${ }^{1}$ INAF - IASF Roma, via del Fosso del Cavaliere 100, 00133 Roma, Italy

e-mail: tavani@iasf-roma.inaf.it

2 Dipartimento di Fisica, Universitá Tor Vergata, via della Ricerca Scientifica 1, 00133 Roma, Italy

${ }^{3}$ Consorzio Interuniversitario Fisica Spaziale (CIFS), villa Gualino, v.le Settimio Severo 63, 10133 Torino, Italy

${ }^{4}$ Dip. Fisica, Università di Trieste, via A. Valerio 2, 34127 Trieste, Italy

5 INFN Trieste, Padriciano 99, 34012 Trieste, Italy

6 INAF - IASF Milano, via E. Bassini 15, 20133 Milano, Italy

7 INFN Roma Tor Vergata, via della Ricerca Scientifica 1, 00133 Roma, Italy

8 INAF - IASF Bologna, via Gobetti 101, 40129 Bologna, Italy

9 ENEA Bologna, via don Fiammelli 2, 40128 Bologna, Italy

10 INFN Roma 1, p.le Aldo Moro 2, 00185 Roma, Italy

11 Dip. Fisica, Università La Sapienza, p.le Aldo Moro 2, 00185

Roma, Italy

12 ENEA Frascati, via Enrico Fermi 45, 00044 Frascati(RM), Italy

13 INFN Pavia, via Bassi 6, 27100 Pavia, Italy

14 ASI Science Data Center, ESRIN, 00044 Frascati(RM), Italy

15 IKI, Moscow, Russia

16 Dipartimento di Fisica, Universitaá di Torino, Torino, Italy

17 Osservatorio Astronomico di Roma, Monte Porzio Catone, Italy

18 Agenzia Spaziale Italiana, viale Liegi 26, 00198 Roma, Italy

19 Carlo Gavazzi Space, via Gallarate 139, 20151 Milano, Italy

20 Thales Alenia Space (formerly Laben), S.S. Padana Superiore 290, 20090 Vimodrone, Milano, Italy

${ }^{21}$ Rheinmetall Italia S.p.A. B.U. Spazio - Contraves, via Affile 102, 00131 Roma, Italy

22 Telespazio, via Tiburtina 965, Roma, Italy

23 Media Lario Technologies, Pascolo, 23842 Bosisio Parini (Lecco), Italy

${ }^{24}$ CNR, IMIP, Montelibretti (Roma), Italy

25 formerly at Carlo Gavazzi Space 